\title{
Cyclophosphamide enhances the antitumor potency of GITR engagement by increasing oligoclonal cytotoxic T cell fitness
}

\author{
Daniel Hirschhorn, ${ }^{1,2}$ Allison Betof Warner, ${ }^{1,2,3}$ Rachana Maniyar, ${ }^{1,2}$ Andrew Chow, ${ }^{1,2,3}$ \\ Levi M.B. Mangarin, ${ }^{1,2}$ Adam D. Cohen, ${ }^{1}$ Linda Hamadene, ${ }^{1,2}$ Gabrielle A. Rizzuto, ${ }^{1,4}$ Sadna Budhu, ${ }^{1,2}$ \\ Nathan Suek, ${ }^{1,2}$ Cailian Liu, ${ }^{1,2}$ Alan N. Houghton, ${ }^{1,2}$ Taha Merghoub, ${ }^{1,2,3}$ and Jedd D. Wolchol1,2,3 \\ 'Swim Across America and Ludwig Collaborative Laboratory, Immunology Program, Parker Institute for Cancer \\ Immunotherapy, and ${ }^{2}$ Human Oncology and Pathogenesis Program, Memorial Sloan Kettering Cancer Center (MSKCC), \\ New York, New York, USA. ${ }^{3}$ Weill Cornell Medical College, New York, New York, USA. ${ }^{4}$ Department of Pathology, University \\ of California, San Francisco, San Francisco, California, USA.
}

Only a subset of cancer patients responds to checkpoint blockade inhibition in the clinic. Strategies to overcome resistance are promising areas of investigation. Targeting glucocorticoid-induced tumor necrosis factor receptor-related protein (GITR) has shown efficacy in preclinical models, but GITR engagement is ineffective in controlling advanced, poorly immunogenic tumors, such as B16 melanoma, and has not yielded benefit in clinical trials. The alkylating agent cyclophosphamide (CTX) depletes regulatory T cells (Tregs), expands tumor-specific effector T cells (Teffs) via homeostatic proliferation, and induces immunogenic cell death. GITR agonism has an inhibitory effect on Tregs and activates Teffs. We therefore hypothesized that CTX and GITR agonism would promote effective antitumor immunity. Here we show that the combination of CTX and GITR agonism controlled tumor growth in clinically relevant mouse models. Mechanistically, we show that the combination therapy caused tumor cell death, clonal expansion of highly active CD8 T cells, and depletion of Tregs by activation-induced cell death. Control of tumor growth was associated with the presence of an expanded population of highly activated, tumor-infiltrating, oligoclonal CD8 ${ }^{+} T$ cells that led to a diminished TCR repertoire. Our studies show that the combination of CTX and GITR agonism is a rational chemoimmunotherapeutic approach that warrants further clinical investigation.

Authorship note: $\mathrm{DH}$ and $\mathrm{ABW}$ are co-first authors. TM and JDW contributed equally to this work.

Conflict of interest: See the online supplement; supplemental material available online with this article; https://doi.org/10.1172/jci. insight.151035DS1.

Copyright: (ㄷ) 2021, Hirschhorn et al. This is an open access article published under the terms of the Creative Commons Attribution 4.0 International License.

Submitted: May 4, 2021 Accepted: September 2, 2021 Published: October 22, 2021

Reference information: /Cl Insight. 2021;6(20):e151035.

https://doi.org/10.1172/jci. insight.151035.

\section{Introduction}

Immune checkpoint blockade is increasingly used to treat a variety of malignancies, but primary and secondary resistance to the FDA-approved cytotoxic T lymphocyte-associated protein 4 (CTLA-4) and programmed cell death protein/ligand 1 (PD-1/PD-L1) inhibitors limit the clinical benefit of these agents (1, 2). One mechanism of both primary and secondary resistance is the presence of $\mathrm{CD} 4^{+} \mathrm{Foxp} 3^{+}$regulatory $\mathrm{T}$ cells (Tregs) in the tumor microenvironment (3). In healthy individuals, Tregs promote self-tolerance and maintain immune homeostasis during stimulation by foreign antigens. Tumors co-opt this mechanism and recruit Tregs that suppress antitumor immune responses in mice (4). Extensive Treg infiltration is also associated with poor prognosis in solid human tumors (5). It is thus reasonable to deduce that therapeutic depletion of Tregs while simultaneously maintaining or enhancing the presence of $\mathrm{CD} 8^{+}$effector $\mathrm{T}$ cells (Teffs) via engagement of costimulatory receptors can overcome resistance to checkpoint blockade.

Glucocorticoid-induced tumor necrosis factor receptor-related protein (GITR), a member of the tumor necrosis factor receptor family, is constitutively expressed at high levels by Tregs and is upregulated when Teffs are activated (6). Engagement of GITR on Tregs inhibits the Foxp3-dependent suppressive lineage commitment, thereby limiting Treg functionality $(7,8)$. GITR engagement provides a costimulatory signal that expands and activates conventional Teffs (9). Thus, GITR agonism increases the ratio of Teff/Treg and promotes antitumor immunity in murine models $(6,7,10)$. We have previously demonstrated that a monoclonal agonist GITR antibody promotes tumor rejection in preclinical models (11-14). However, in clinical trials, GITR agonism as a monotherapy has failed to effectively control tumor growth, likely due to 
insufficient Treg modulation and exhaustion of Teffs $(15,16)$. To address T cell exhaustion, previous work by our group combined GITR agonist antibodies with anti-PD-1 in the B16 melanoma model, demonstrating effective tumor control (16). ClinicalTrials.gov NCT02628574 was designed to evaluate GITR agonism alone or in combination with anti-PD-1 therapy or gemcitabine, but data are not yet available.

Another active area of investigation is the strategy of combining chemotherapy and immune checkpoint blockade, in part due to the potentially additive or even synergistic effects of these agents (17). Cytotoxic agents can stimulate antitumor responses by killing tumor cells to release potentially antigenic debris, increasing immune infiltration, depleting Tregs and myeloid-derived suppressor cells, and enabling homeostatic proliferation of antigen-specific Teffs (18-22).

Cyclophosphamide (CTX) is an alkylating agent with direct antitumor activity that can increase antigen cross-presentation and cross-priming (23) and also suppress Tregs (24-26). Lymphodepletion induced by CTX is followed by a recovery phase, allowing for expansion of tumor-specific Teffs (22). During the recovery phase, proinflammatory cytokines, including interferons, are upregulated and can promote antitumor activity $(27,28)$.

Cytotoxic agents such as CTX cause DNA damage and immunogenic cell death of tumor cells $(29,30)$. The surviving cells can accumulate mutations promoting the development of neoantigens, which can trigger antitumor $\mathrm{T}$ cells. One report demonstrates that treating poor responders to immune checkpoint inhibitors with cytotoxic chemotherapy induces subclonal neoantigens (31). Additionally, gliomas that recur after treatment with temozolomide (a DNA-alkylating agent) contain several mutations with neoantigen potential (32, 33). Therefore, CTX could improve an antitumor response by promoting the development of neoantigens.

Effective immune control of tumor growth is likely a result of Treg suppression coupled with expansion, infiltration, and activation of $\mathrm{CD}^{+} \mathrm{Teffs}$, which have the capacity to kill tumor cells upon recognition of specific antigens by the T cell receptor (TCR). High-throughput TCR sequencing allows quantification of $\mathrm{T}$ cell diversity and identification of tumor-reactive T cell clonotypes (34). High TCR clonality correlates with oligoclonal expansion and improved immunotherapy efficacy in melanoma and other solid tumors (35-38). T cell clonality both in the periphery and within the tumor might certainly be altered as $\mathrm{T}$ cells recover after CTX-induced lymphodepletion.

In this study we hypothesize that administration of CTX prior to agonist anti-GITR antibodies would generate a potent antitumor response because the GITR activating signal would be delivered to Teffs during the recovery phase from lymphodepletion.

We demonstrate that CTX, in combination with anti-GITR, promoted durable antitumor responses in clinically relevant tumor models, such as MPC-11 plasmacytoma and the poorly immunogenic B16 melanoma. This combination induced tumor cell death and potently suppressed Tregs, thereby resulting in highly activated $\mathrm{CD} 8^{+}$Teffs and increased oligoclonal cytotoxic T cell fitness.

\section{Results}

Dose-dependent modulation of GITR expression in T cells by CTX. CTX causes acute lymphodepletion followed by homeostatic proliferation. We administered increasing doses of CTX ranging from 30-250 mg/kg intraperitoneally to mice and tested overall cellular composition in the spleen at different time points (Figure 1A). Low doses of CTX (30 and $75 \mathrm{mg} / \mathrm{kg}$ ) induced mild splenocyte depletion, whereas higher doses (150 and 250 mg/kg) promoted robust depletion of splenocytes, with a nadir at day 4 after treatment. Interestingly, at the $250 \mathrm{mg} / \mathrm{kg}$ dose, we observed an increased rebound in total splenic cellularity with approximately $50 \%$ more cells than pretreatment at day 10 . We chose the $250 \mathrm{mg} / \mathrm{kg}$ dose for further experimentation because of this most profound change in splenic cellularity. At the $250 \mathrm{mg} / \mathrm{kg}$ dose, $\mathrm{T}$ cells in the spleen $\left(\mathrm{CD} 8^{+} \mathrm{CD} 4^{+} \mathrm{Foxp} 3^{-}\right.$conventional $\mathrm{T}$ [Tconv] cells and $\mathrm{CD}^{+}{ }^{+} \mathrm{Foxp}^{+}$Tregs) showed similar kinetics to total splenocytes with a nadir at day 4 and recovery to baseline numbers at day 7 (Figure 1B). Increased levels of the proliferation marker Ki67 were found in all $\mathrm{T}$ cell subsets tested on day 11, about 1 week after the nadir, indicating extensive homeostatic proliferation (Figure 1C). Given that homeostatic proliferation promotes $\mathrm{T}$ cell activation, we next tested whether CTX administration modulates the expression of activation markers, such as GITR. A modest modulation of GITR expression on $\mathrm{CD}^{+}$Foxp3 $^{-}$Tconv and CD8 ${ }^{+} \mathrm{T}$ cells was observed, with greater effects on CD4 ${ }^{+}$Foxp3 ${ }^{+}$Tregs (Figure 1, D-F). Closer examination of Tconv cells revealed that GITR was upregulated predominantly by the Ki67 $7^{+}$fraction of $\mathrm{CD}^{+}$and $\mathrm{CD}^{+}$Tconv cells, consistent with an activated phenotype in the homeostatically proliferating cells (Figure 1, D and E). Both the Ki67 ${ }^{+}$and $\mathrm{Ki}^{-} 7^{-}$fractions of Tregs showed similar fluctuations in GITR expression after CTX treatment (Figure 1F). In summary, a CTX dose of $250 \mathrm{mg} / \mathrm{kg}$ caused lymphopenia that was followed by homeostatic proliferation and changes in the level of GITR expression on Tconv cells and Tregs. 
A
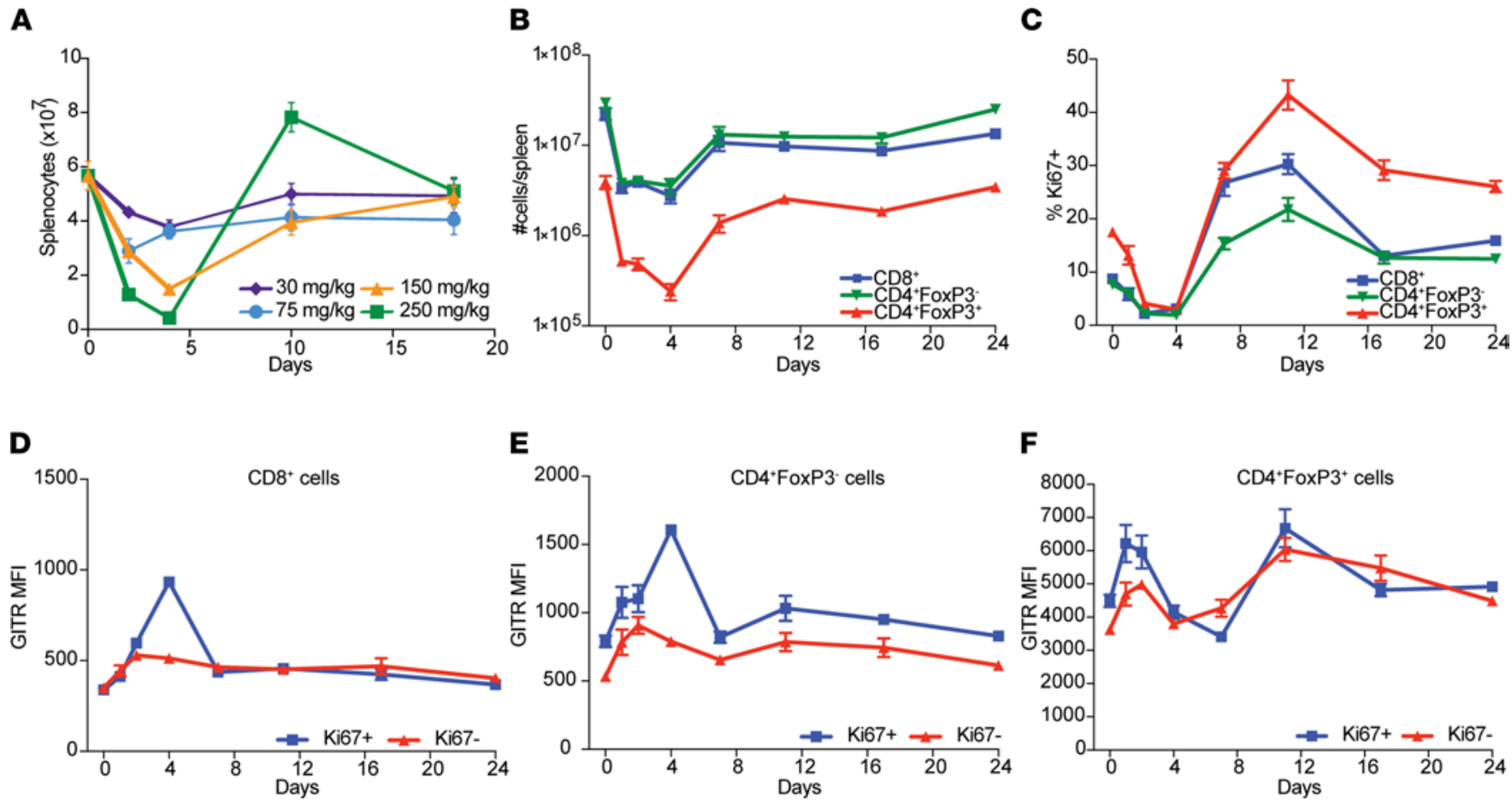

Figure 1. CTX modulates GITR expression in T cell populations in a dose-dependent manner. (A) Cohorts of 5 mice were treated with $30,75,150$, and 250 $\mathrm{mg} / \mathrm{kg}$ CTX. Single-cell suspensions were prepared from spleens at the depicted days and analyzed for total cellularity by flow cytometry. Number of cells plotted against day post-CTX. (B-F) Cohorts of 5 mice were treated with $250 \mathrm{mg} / \mathrm{kg}$ of CTX. Single-cell suspensions were prepared from the spleens at depicted days and analyzed by flow cytometry. (B) Number of splenic CD8 ${ }^{+}, \mathrm{CD}^{+} \mathrm{FoxP3}^{-}$, and $\mathrm{CD} 4^{+} \mathrm{FoxP3}{ }^{+} \mathrm{T}$ cells show nadir at day 4 with a homeostatic recovery at day 11. (C) Percentages of $\mathrm{Ki}^{6} 7^{+} \mathrm{CD}^{+}, \mathrm{CD}^{+} \mathrm{FoxP3}^{-}$, and $\mathrm{CD} 4^{+} \mathrm{FoxP3}^{+} \mathrm{T}$ cells peak at 11 days. (D) GITR MFI on Ki67 ${ }^{+}$and Ki67- $\mathrm{CD} 8^{+}$gate. (E) GITR MFI on Ki67 ${ }^{+}$and Ki67- $\mathrm{CD}^{+}{ }^{+} \mathrm{FoxP3}^{-}$gate. (F) GITR MFI on $\mathrm{Ki}^{-} 7^{+}$and $\mathrm{Ki}^{-} 7^{-} \mathrm{CD}^{+}{ }^{+} \mathrm{FoxP3} 3^{+}$gate. Symbols represent the average of 5 mice at a given day $\pm \mathrm{SEM}$. This experiment was repeated at least 3 times with similar results.

The combination of CTX and anti-GITR effectively controls B16 melanoma progression. Given that CTX modulates GITR expression on T cell subsets, promotes homeostatic proliferation of T cells, and depletes Tregs, we hypothesized that CTX administration could enhance the antitumor properties of GITR agonist antibodies. We treated established B16 melanoma tumors with $250 \mathrm{mg} / \mathrm{kg}$ CTX on day 8 followed by the GITR agonist antibody DTA-1 on day 9 (Figure 2A). Tumor growth of B16 melanoma was significantly delayed with $\mathrm{CTX}+$ anti-GITR agonist combination therapy, and several mice experienced tumor regression (Figure 2B). The combination therapy significantly prolonged survival in this model (Figure $2 \mathrm{C}$ ). To further demonstrate the versatility of the combination therapy, we treated mice bearing MPC-11 plasmacytoma and observed similar effects (Figure 2, D and E). While CTX showed partial antitumor effects in the models, only the combination therapy with the GITR agonist antibody led to significant tumor regression.

Superior synergy of GITR agonism and CTX compared with other cytotoxic agents. Once we established that the antitumor activity of GITR engagement was enhanced after a single dose of $250 \mathrm{mg} / \mathrm{kg}$ CTX, we evaluated whether other cytotoxic agents have similar effects (Figure 3A). Gemcitabine (gem) was selected because of its immunomodulatory properties, particularly on myeloid-derived suppressor cells (21). Similar to CTX, gem causes an initial delay in tumor growth, but unlike CTX, administration of gem did not increase the efficacy of GITR agonism (Figure 3, B and C). Whole-body irradiation induces lymphopenia (39) but does not directly affect the growth of radioresistant B16 (40). We then investigated the effects of total-body irradiation, or lower dose CTX, in combination with anti-GITR (Figure 3D). Tumor rejection was only observed with the combination treatment of anti-GITR and high-dose CTX (Figure 3, E and F). Therefore, alkylating agents are conducive to enhancing the antitumor properties of anti-GITR antibodies over other cytolytic agents tested.

High-dose CTX causes tumor cell death and induces in situ vaccination. Once we established that CTX was a superior combination agent, we hypothesized that CTX-induced tumor cell death was contributing to the efficacy of anti-GITR and high-dose CTX. To test this, we measured the proliferation of $\mathrm{CD} 8^{+} \mathrm{T}$ cells by transferring CFSE-labeled CD8 ${ }^{+} \mathrm{T}$ cells recognizing the melanoma antigen Pmel (41), after CTX administration (Figure 4A). We observed substantial proliferation of tumor-specific $\mathrm{T}$ cells in the lymph nodes 
A

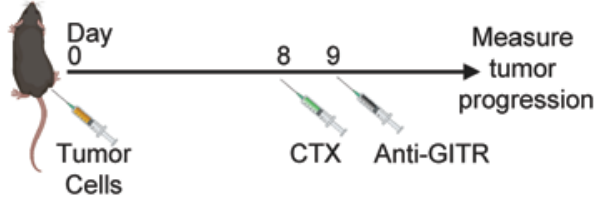

(B16 or MPC-11)

B
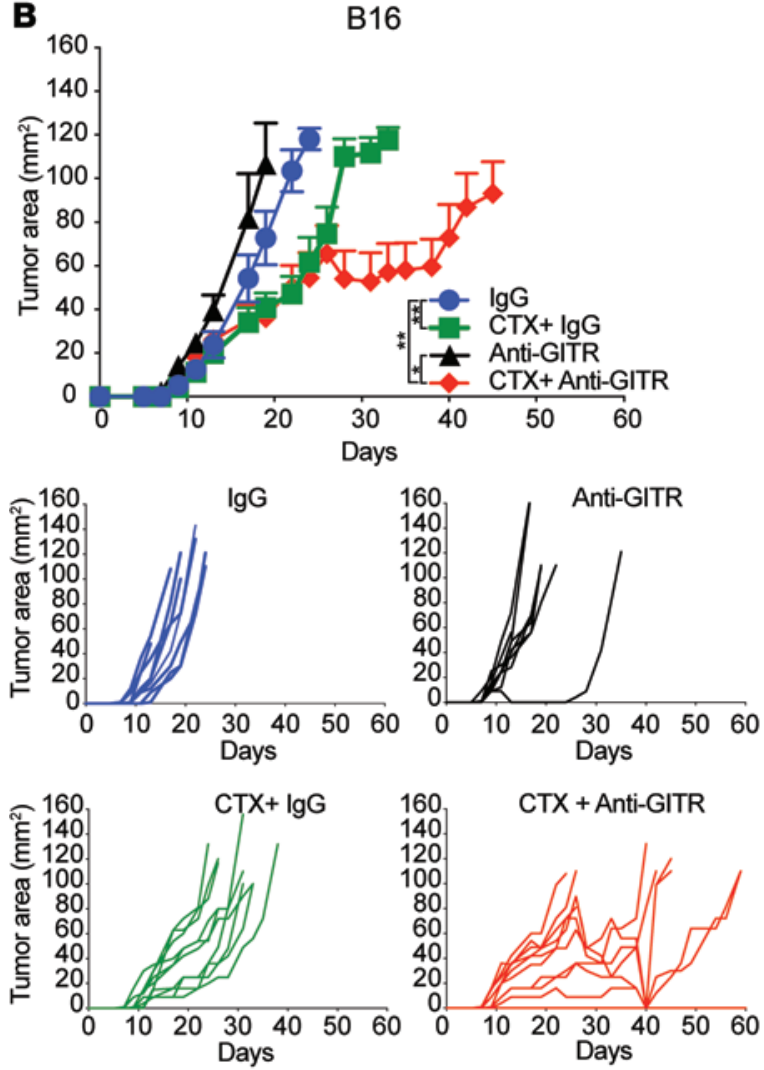

C

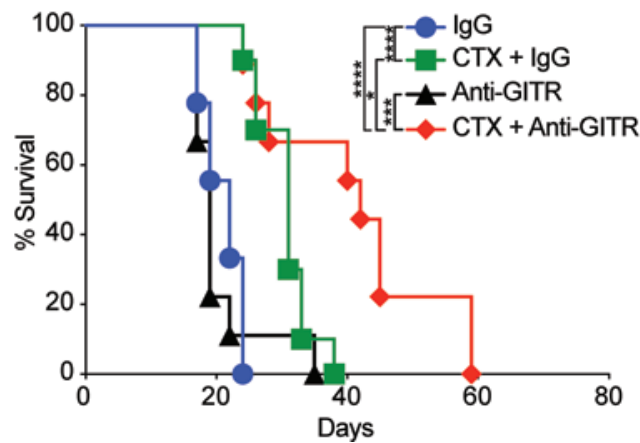

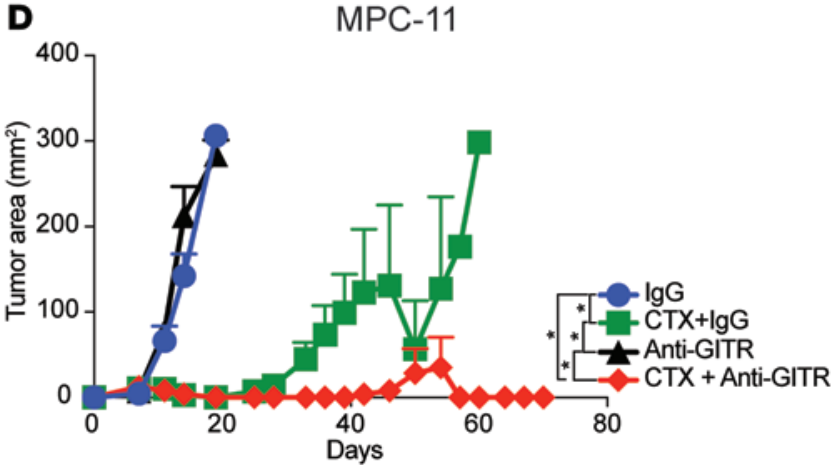
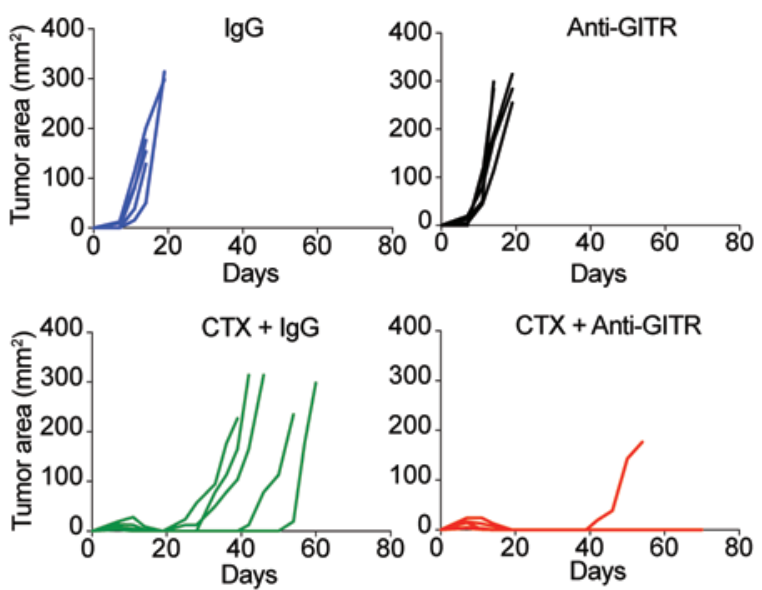

E

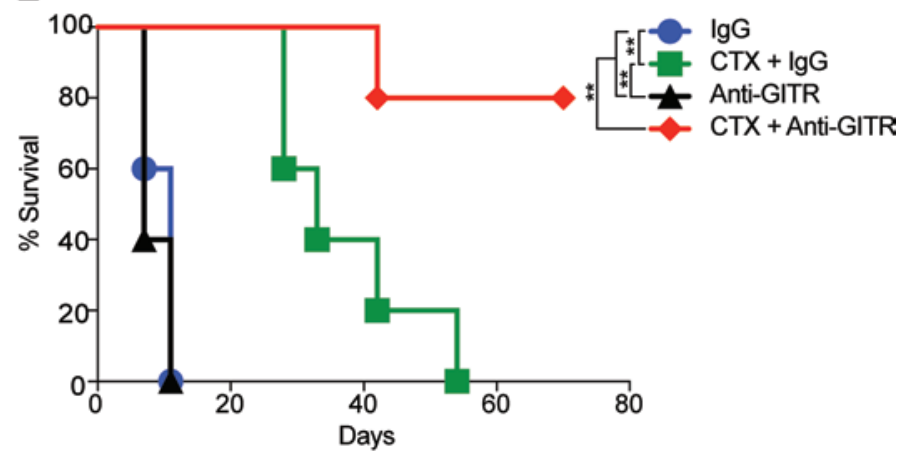

Figure 2. CTX synergizes with anti-GITR to promote potent tumor immunity. (A) Experiment schema: Cohorts of 10 mice were implanted intradermally in the flank with tumor cells (B and C) B16 melanoma and (D and E) MPC-11 plasmacytoma. On day 8, once tumors were palpable, CTX or PBS was injected. On day 9, mice were treated with anti-GITR antibody or rat IgG. Tumor size was measured 3 times weekly. (B) B16 melanoma tumor growth curves (top); growth curves of individual mice over time by treatment group (bottom). Only groups treated with combination therapy demonstrated regression. (C) Kaplan-Meier survival plot demonstrating improved overall survival in the B16-bearing mice treated with CTX + anti-GITR. (D) MPC-11 plasmacytoma tumor growth curves (top); growth curves of individual mice over time by treatment group (bottom). (E) Kaplan-Meier survival plot demonstrating improved overall survival in the MPC-11-bearing mice treated with CTX + anti-GITR. Symbols represent average tumor area \pm SEM. Two-way ANOVA was used followed by a Tukey's multiple-comparison test for tumor growth curve comparisons. Log-rank (Mantel-Cox) test was used for Kaplan-Meier survival curve comparisons. ${ }^{*} P<0.05$, ${ }^{* *} P<0.01$, ${ }^{* *} P<0.001,{ }^{* * *} P<0.0001$. Experiments were repeated twice with similar responses. 
A

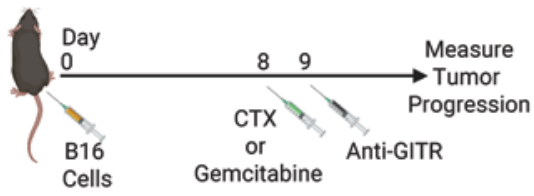

B

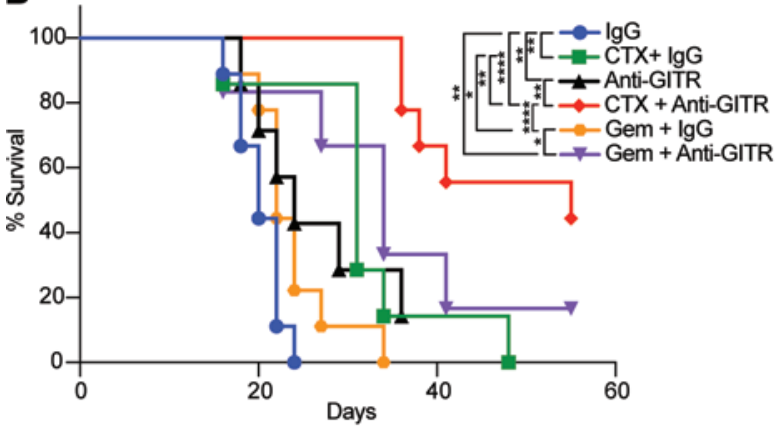

C
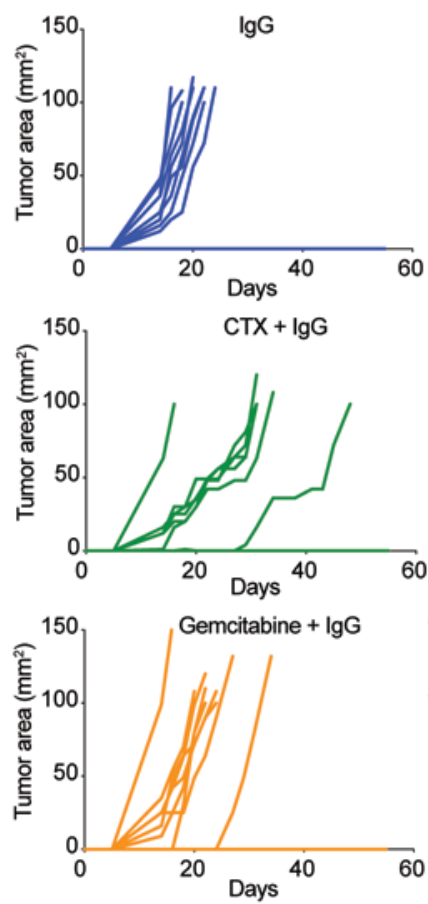
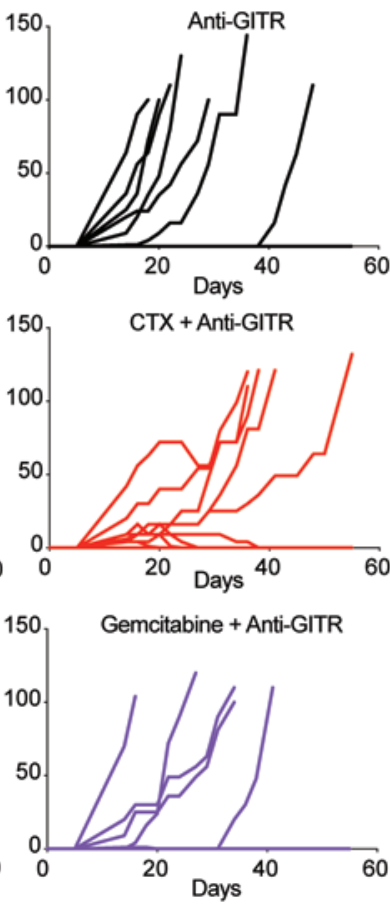

D

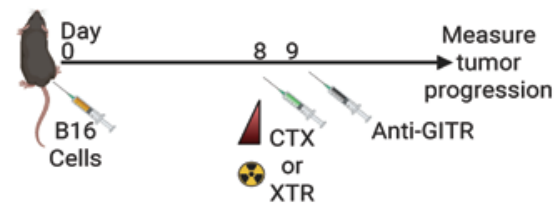

E

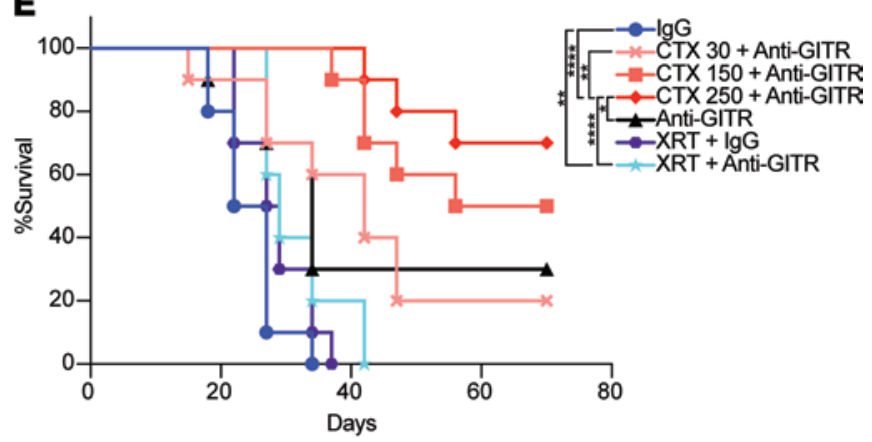

$\mathbf{F}$
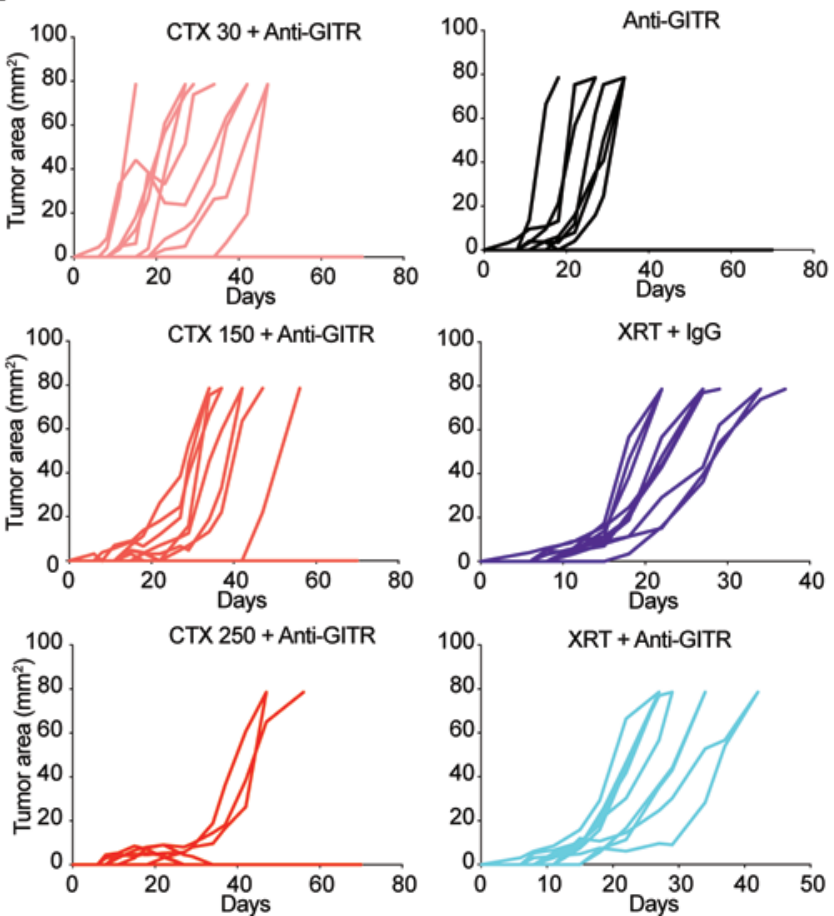

Figure 3. Superior synergy of GITR agonism and CTX compared with other cytotoxic agents. (A) Experiment schema for B and C. Cohorts of 10 mice were implanted intradermally in the flank with B16 melanoma. On day 8, CTX or gemcitabine (Gem) was injected i.p. On day 9, mice were injected with DTA-1 (anti-GITR) or rat IgG. (B) Kaplan-Meier survival curves showing improved survival in the CTX + anti-CITR combination treatment groups. (C) Tumor growth curves of individual mice per treatment group. (D) Experiment schema for $\mathbf{E}$ and $\mathbf{F}$. Cohorts of 10 mice were implanted intradermally with B16 melanoma. On day 8, 30, 150, or $250 \mathrm{mg} / \mathrm{kg}$ CTX was injected i.p., or mice received 6 Gy of total-body irradiation. On day 9, mice were treated with DTA-1 (anti-GITR) or rat IgG. (E) Kaplan-Meier survival curves showing improved survival only in mice treated with a combination of CTX and anti-GITR. (F) Tumor growth curves of individual mice per treatment. Log-rank (Mantel-Cox) test was used for Kaplan-Meier survival curve comparisons. ${ }^{*} P<0.05,{ }^{* *} P<0.01,{ }^{* * * *} P<0.0001$. Experiments were repeated at least twice with similar results.

draining the tumor (TDLNs) but not in the contralateral non-TDLNs. Moreover, further increased proliferation of Pmel-1 T cells was observed in TDLNs compared with non-TDLNs when anti-GITR was administered (Figure 4B). To demonstrate whether the effect was due to CTX alone or the tumor must be present to prime the immune response, we administered CTX either before or after tumor implantation (Figure 4C). Only CTX administration after tumor challenge synergized with GITR agonism in promoting tumor control and improving overall survival (Figure 4, D and E), indicating that tumor cells were essential for the 
A

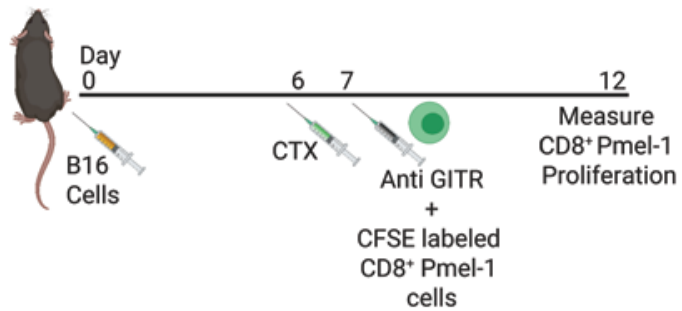

B

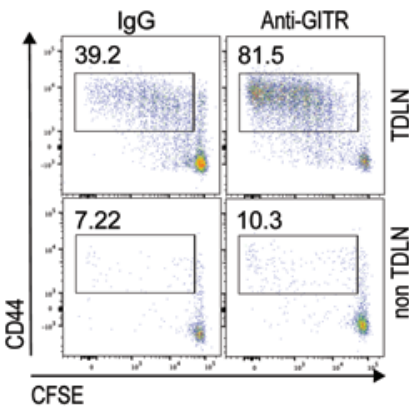

C

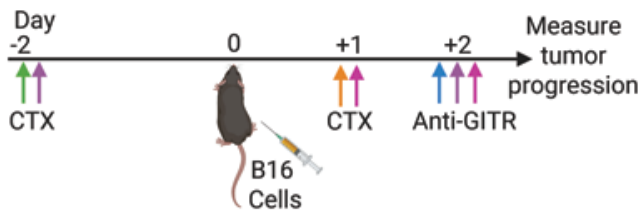

D
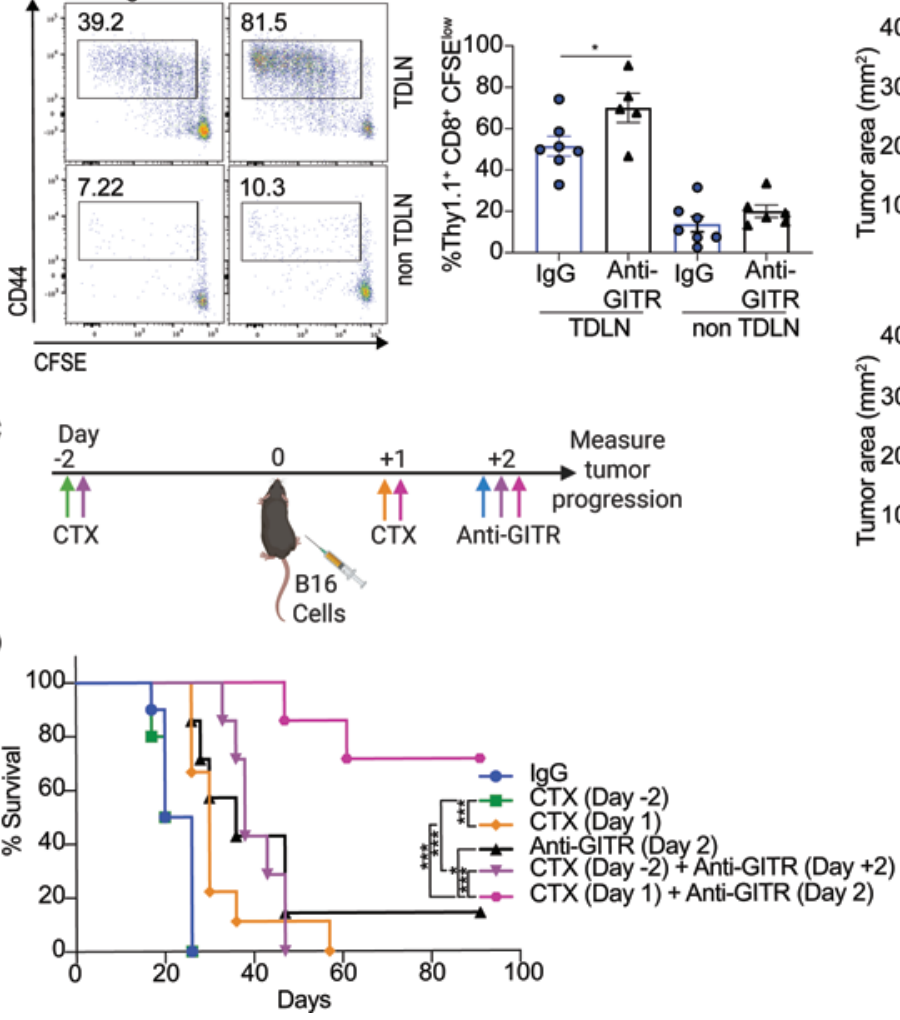
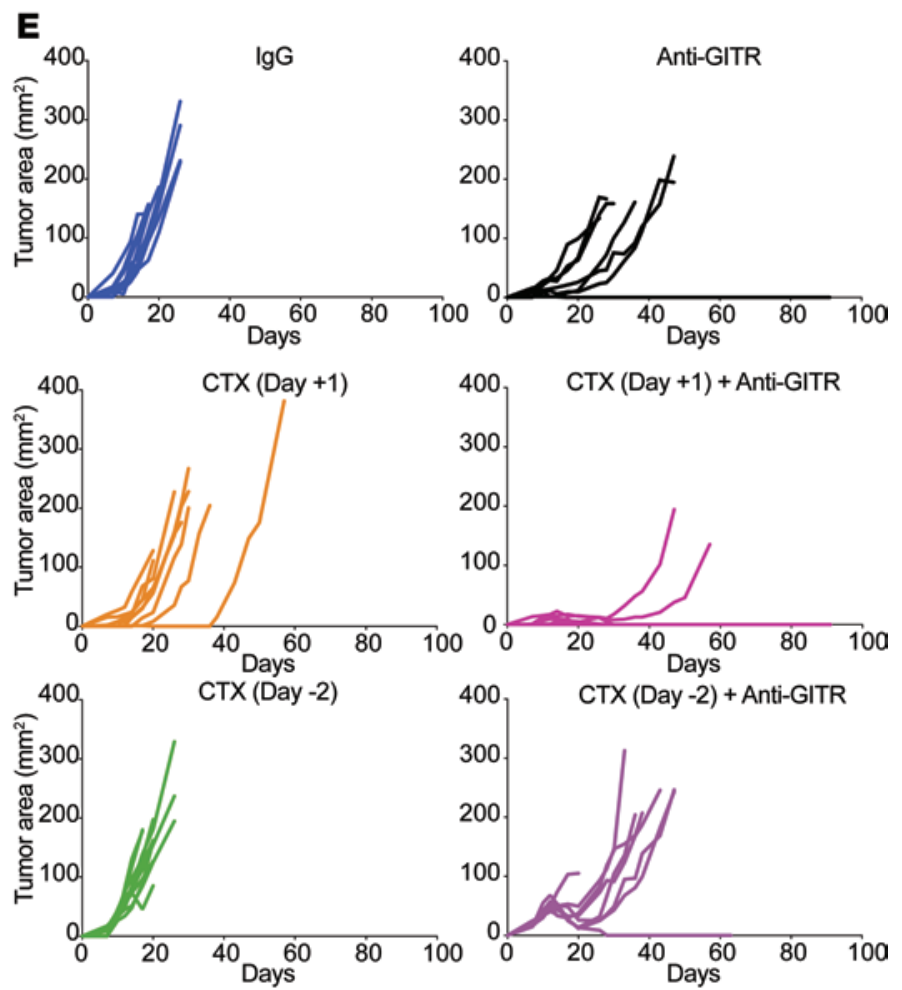

Figure 4. High-dose CTX causes tumor cell death and induces in situ vaccination. (A) Experiment schema: Thy1.2 mice were injected with B16. On day 6 , groups mice $(n \geq 5)$ were treated with CTX or PBS. On day 7, all mice received CFSE-labeled CD8 ${ }^{+}$pmel-1 T cells (Thy1.1) and anti-GITR or rat IgG. On day 12, mice were sacrificed, a single-cell suspension was prepared from inguinal tumor-draining lymph nodes (TDLNs) or contralateral lymph nodes (non-TDLNs), and proliferation was analyzed by flow cytometry. (B) Representative flow plot (left) of proliferating antigen-experienced cells gated on DAPI', CD8 ${ }^{+}$, and Thy1.1 $1^{+}$CFSE ${ }^{10}$. Bars (right) represent the average of 5-7 mice \pm SEM. Symbols represent individual mice and lines represent averages \pm SEM. Unpaired 2 -tailed Student's $t$ test was used to compare IgC and anti-GITR groups. ${ }^{*} P<0.05$. (C) Experiment schema: Cohorts of mice $(n=10)$ were injected with CTX at day -2 or +1 relative to B16 tumor implant. Some cohorts were injected with DTA-1 at day 2 after tumor challenge. (D) Kaplan-Meier survival curves demonstrating improved survival in the group treated with a combination therapy after tumor implant. Log-rank (Mantel-Cox) test was used to compare Kaplan-Meier survival curves. (E) Tumor growth curves of individual mice per treatment group demonstrating a regression only when treated with CTX post tumor implant in combination with anti-GITR. ${ }^{*} P<0.05$, ${ }^{* *} P<0.001$. Experiments were repeated at least twice with similar results.

observed response. Overall, these results demonstrate that high-dose CTX combined with anti-GITR antibodies regresses tumors, and this is associated with antigen-specific $\mathrm{CD} 8^{+} \mathrm{T}$ cell proliferation in the TDLNs. This result warrants further investigation into the effects of additional cytotoxic agents in combination with GITR agonism and whether they phenocopy the effects observed with CTX.

The combination of CTX and anti-GITR decreases intratumor Tregs and increases the ratio of $C D 8^{+} T$ cells to Tregs. Recruitment of $\mathrm{CD}^{+}{ }^{+} \mathrm{Foxp}^{+}$Tregs to the tumor bed is an important mechanism by which tumors evade immune surveillance (4). GITR engagement can downregulate the quantity and function of Tregs in tumors $(12,14,16,42)$. Moreover, CTX administration suppresses Tregs in tumors and the periphery (26). To determine whether the combination of CTX and GITR agonism affects intratumor Tregs, we first performed flow cytometry (Figure 5A) and observed reduced absolute numbers of Tregs in mice treated with the combination therapy compared with controls (Figure 5B). It is known that an elevated ratio of $\mathrm{CD} 8^{+} \mathrm{T}$ cells/Tregs is predictive of immunotherapy efficacy (43). Upon closer examination, we found that the ratio 
A

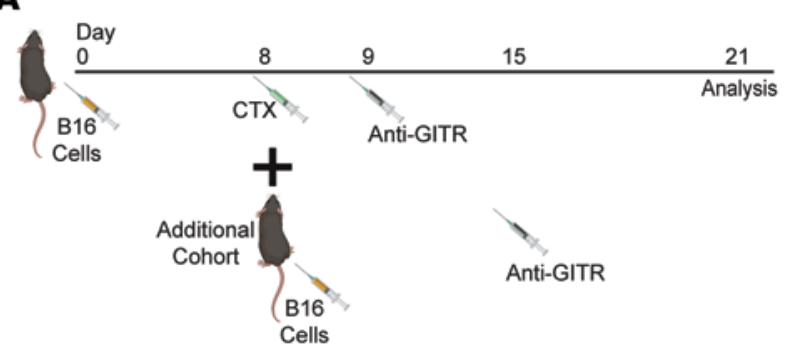

D

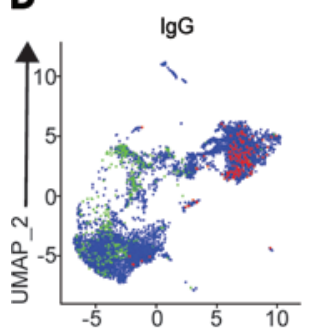

UMAP_1

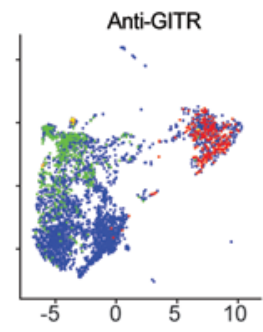

B

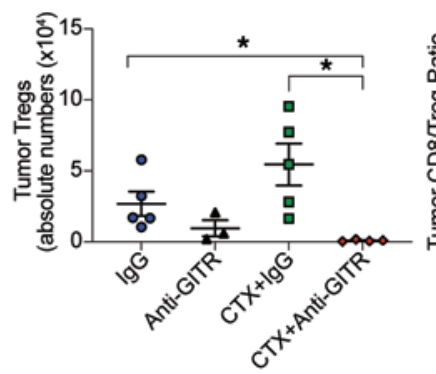

C

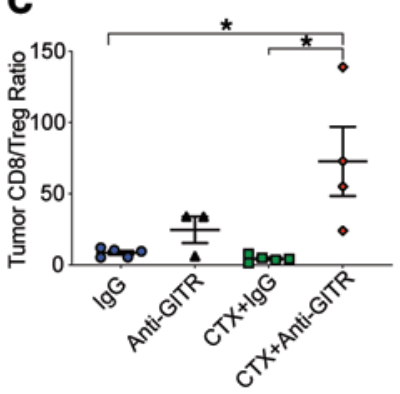

E
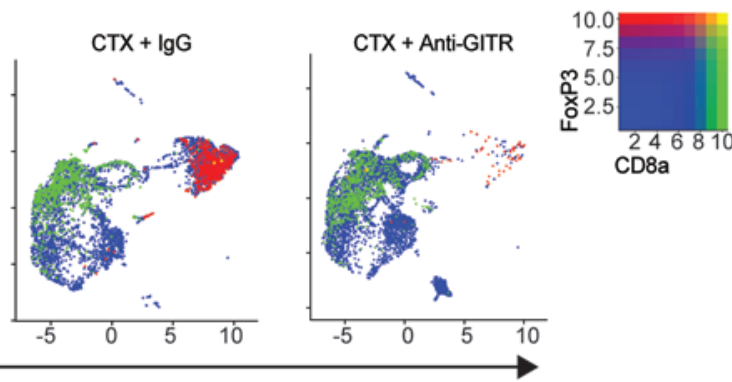

$\mathbf{F}$
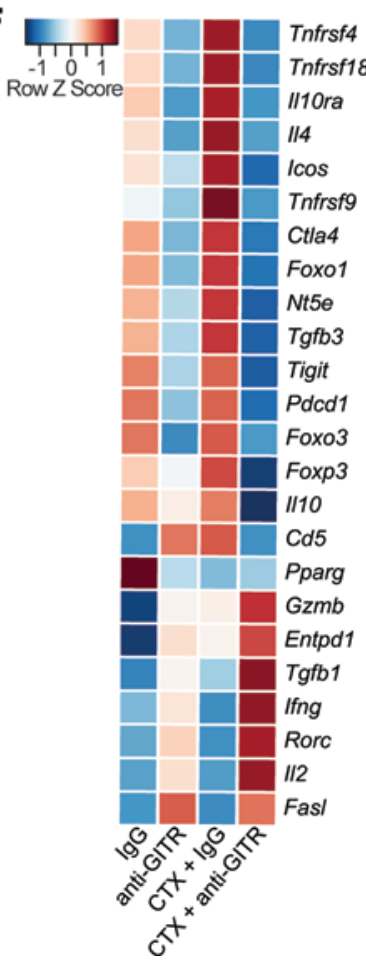

Figure 5. Combination therapy decreases intratumor Tregs and increases CD8/Treg ratio. Mice were implanted with B16 on day 0 and then treated with CTX on day 8 and anti-GITR or control IgG on day 9. Additional cohorts of mice were implanted with B16 on day 8; on day 15 mice were treated with anti-GITR or IgG. After 1 week, all cohorts of mice were sacrificed, and single-cell suspensions were prepared from tumors. Asynchronous B16 challenge was needed for the CTX groups a week apart given the lack of immune infiltrates caused by chemotherapy treatment. (A) Experiment schema. (B) Absolute number of Tregs per gram of tumor per treatment group demonstrating a reduction in Tregs in the combination treatment group. (C) Ratio of total CD $8^{+} T$ cells to Tregs measured by flow cytometry demonstrating an increase in the combination treatment group. (D) scRNA-Seq of CD5+ sorted T cells pooled from 5 mice for each treatment. UMAP plots for each treatment are shown for the expression of CD8a and Foxp3, demonstrating a reduction in Foxp $3^{+}$cells in the combination treated group. (E) Representative flow cytometry plots of Foxp3 versus Live/Dead viability dye from tumor cells. Cells were pregated on CD4 ${ }^{+} T$ cells (left). Percentages of Foxp3 $3^{+}$that are Dead ${ }^{+}$(right). (F) Differential analysis of gene expression by Tregs on selected groups analyzed by single-cell sequencing. Ordinary 1-way ANOVA with a Tukey's multiple-comparison test was used. Symbols represent individual mice and lines represent averages $\pm \mathrm{SEM} .{ }^{*} P<0.05$, ${ }^{* * * *} P<0.0001$. Flow cytometry experiments were repeated at least twice with similar results. Gzmb, Granzyme B; FasL, Fas ligand.

of $\mathrm{CD}^{+} \mathrm{T}$ cells/Tregs in the tumors was several-fold higher with the combination therapy than with either monotherapy (Figure 5C).

We further evaluated intratumor T cells by single-cell RNA sequencing (scRNA-Seq). T cells were isolated based on CD5 expression by FACS from B16 tumors of treated mice or controls. After appropriate quality controls, the cells were subjected to single-cell sequencing using the 10x Genomics platform coupled with TCR sequencing. We pooled intratumor T cells from mice treated with IgG, anti-GITR, CTX IgG, and CTX + anti-GITR. We included "hashtag" antibodies to determine intermouse variation. The uniform manifold approximation and projection for dimension reduction (UMAP) plots demonstrate that the combination therapy substantially decreased the number of Tregs and increased CD8 $8^{+} \mathrm{T}$ cells (Figure $5 \mathrm{D}$ and Supplemental Figure 1; supplemental material available online with this article; https://doi.org/10.1172/jci. insight.151035DS1). These data further confirm the changes observed by flow cytometry. 
Next, we investigated how the combination treatment might lead to fewer intratumor Tregs. Immunotherapeutic interventions can reduce the number of intratumor Tregs by a variety of mechanisms, including activation-induced cell death (41). Using flow cytometry to characterize tumor-infiltrating Tregs, we observed that significantly more Tregs underwent cell death in the combination group versus control mice (Figure 5E). These findings indicate that Treg cell death might contribute to the overall efficacy of the combination treatment.

Our laboratory has previously shown that GITR engagement destabilizes Foxp3 and the suppressive Treg lineage (7). Using our single-cell sequencing data, clustering was performed to identify the intratumor Tregs in different treatment groups, and we ran differential expression analyses for selective suppressive and effector genes. As shown in Figure 5F, consistent with our previous observations, GITR agonism decreased the suppressive profile, endowing a $\mathrm{T}$ effector-like signature to Tregs characterized by upregulation of proinflammatory cytokines (IL-2 and IFN- $\gamma$ ) and cytolytic molecules (Gzmb and FasL). Interestingly, CTX alone induced Tregs that showed increased expression of tumor necrosis factor receptor superfamily members, Tnfrsf4 (OX40), Tnfrsf9 (4-1BB), Tnfrsf18 (GITR), and inducible T cell costimulatory molecule, which were recently described as activated Tregs that are selectively associated with poor outcomes in non-small cell lung cancer (44). Importantly, the addition of GITR agonist antibody to CTX profoundly abrogated this activated Treg phenotype. Furthermore, the remaining cells acquired an even higher cytolytic Teff-like phenotype.

Combination of CTX and GITR agonism promotes cytotoxic terminally differentiated CD $8^{+} T$ cells. Given that the combination of CTX and GITR agonist antibody decreased intratumor Tregs and decreased Treg functionality, we next examined whether the combination increased functionality of tumor-infiltrating $\mathrm{CD} 8^{+} \mathrm{T}$ cells. First, we examined the overall landscape of intratumor $\mathrm{CD} 8^{+} \mathrm{T}$ cells by pathway analysis from the scRNASeq, described in detail in Methods. We used unsupervised clustering and then applied a predefined set of genes based on biological priors (see Methods) to define the CD8 cluster. We found that $\mathrm{CD} 8^{+} \mathrm{T}$ cells from the combination therapy group had a signature of higher activation (Figure 6A). Accordingly, scRNA-Seq showed increased transcription of a cytolytic program consisting of upregulation of granzymes, perforin, and FasL with the combination therapy, suggesting a highly activated state (Figure 6B and Supplemental Figure 2). Given the enhanced cytolytic profile with combination therapy, we further characterized the activation state of the $\mathrm{CD}^{+} \mathrm{T}$ cells at the protein level. Flow cytometry from single-cell suspensions prepared from tumors of treated mice demonstrated that intratumor $\mathrm{CD} 8^{+} \mathrm{T}$ cells from mice treated with combination therapy had an enhanced activation and terminal differentiation phenotype characterized by PD- $1^{\text {lo }} C D 25^{\text {hi }} \mathrm{KLRG} 1{ }^{\mathrm{lo}} \mathrm{Blimp} 1^{\mathrm{hi}}$ staining compared with treatment with CTX alone (Figure 6C) (45). Moreover, a subset of tumor-infiltrating $\mathrm{CD}^{+} \mathrm{T}$ cells were Eomes ${ }^{\text {hiT-bet }}{ }^{\text {lo }}$ (Figure 6D). Interestingly, this phenotype is associated with both an antigen-experienced, highly activated state as well as an exhausted/dysfunctional state of these cells (46, 47). To address this complexity, we stimulated single-cell suspensions, derived from tumors of treated mice, ex vivo with PMA/ionomycin and examined cytokine expression. The $\mathrm{CD} 8^{+} \mathrm{T}$ cells treated with combination therapy showed higher levels of TNF- $\alpha$ and IFN- $\gamma$, indicating that they were functional and not exhausted (Figure 6E). In conclusion, we found that the combination of CTX and GITR engagement promoted the expansion of differentiated, highly cytotoxic intratumor $\mathrm{CD} 8^{+} \mathrm{T}$ cells.

Combination of CTX and anti-GITR constricts the TCR repertoire with expansion of highly activated $C D 8^{+} T$ cells. The effects of novel immunotherapy regimens on the TCR repertoire and on the phenotype of tumorreactive clones are still open questions. Immune-perturbing interventions may lead to diversification or clonal expansion of the TCR repertoire (48). To address this, we obtained paired TCR-sequencing data that could be linked to the aforementioned scRNA-Seq data. The advantage of this approach is that TCR identity and phenotype can be simultaneously studied at the single-cell level. First, we asked how the combination of CTX and GITR agonism affects the entire TCR repertoire. Circos plots of VDJ-rearranged TCR segments are shown in Figure 7A. As expected for settings where there is expansion of antigen-specific T cells, combination therapy yielded a population of $\mathrm{CD}^{+}$cells that showed reduced TCR diversity (i.e., increased clonal expansion). These results were supported by calculating a Shannon-Weiner evenness diversity score, which demonstrated increased clonal expansion with the combination therapy (Figure 7B). Examination of the top 10 most frequent clones in the CTX + anti-GITR treatment group showed a shift toward expansion of activated/ exhausted $\mathrm{CD}^{+} \mathrm{T}$ cells in the defined UMAP (Figure 8, A and B; Supplemental Figure 3; and Supplemental Tables 1 and 2). We assigned a definition of $\mathrm{T}$ cell clusters supervised on lineage and functional markers on the aggregate $(49,50)$ (Figure $8 \mathrm{~A}$ and Supplemental Figure 4; see Methods). Interestingly, many of the top 10 most frequent clones in the IgG and CTX plus IgG groups were Foxp $3^{+}$Tregs, indicating that an important role of anti-GITR therapy is eliminating the tumor- and CTX-associated expansion of Tregs. 
A
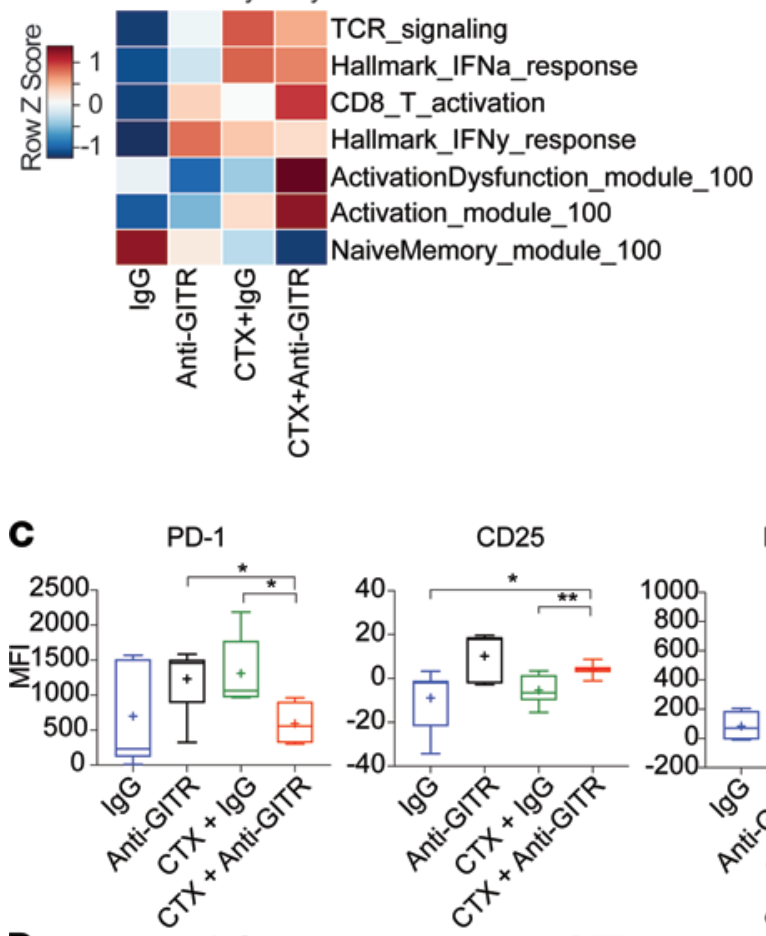

D

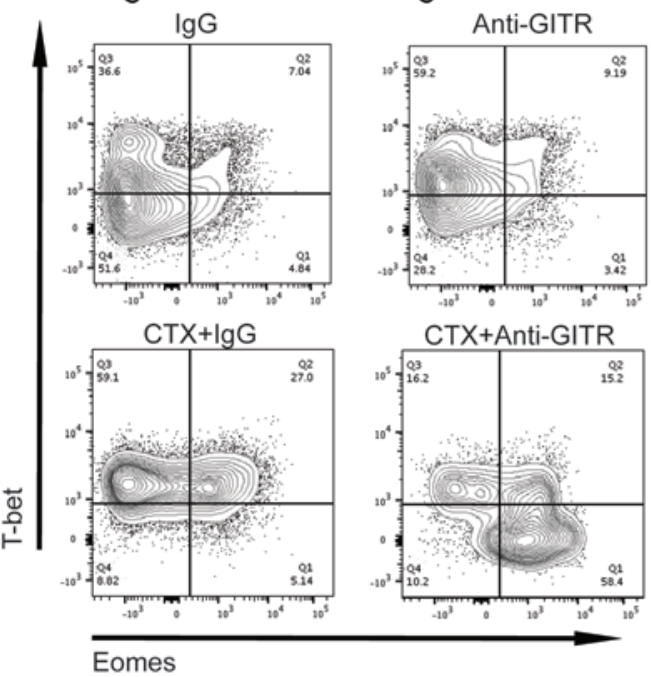

E
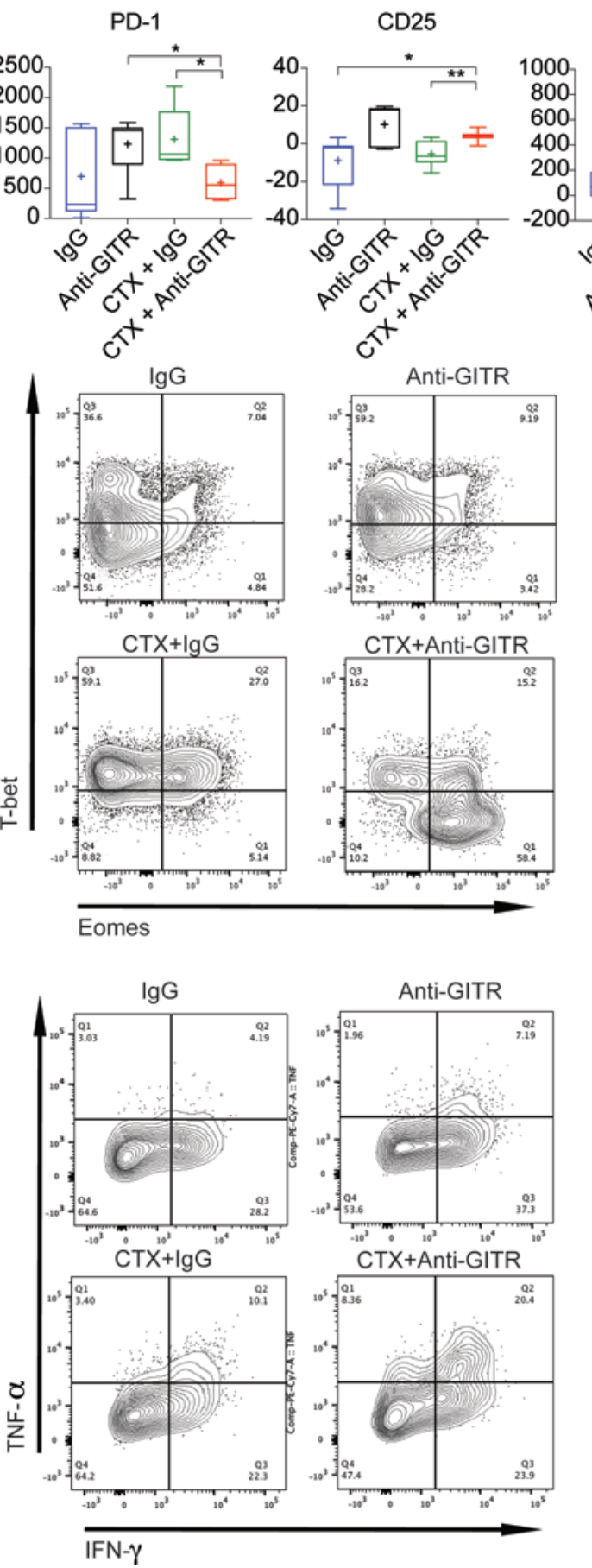

B
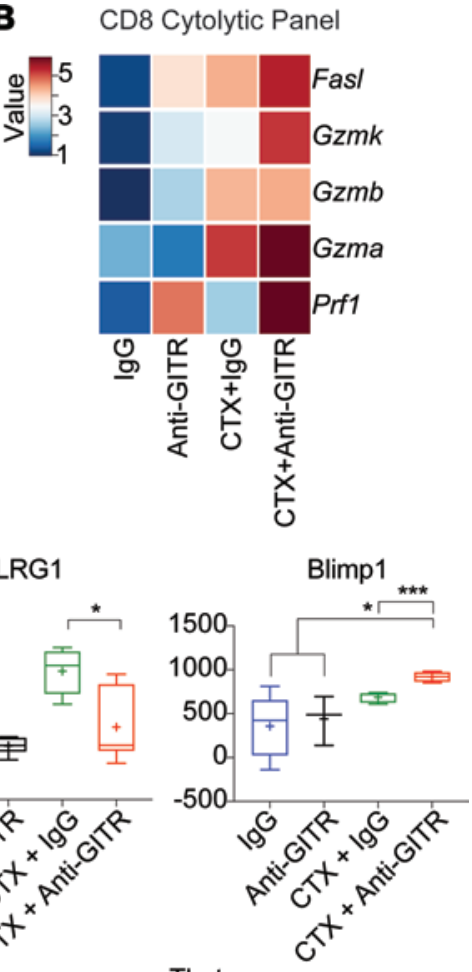

Tbet

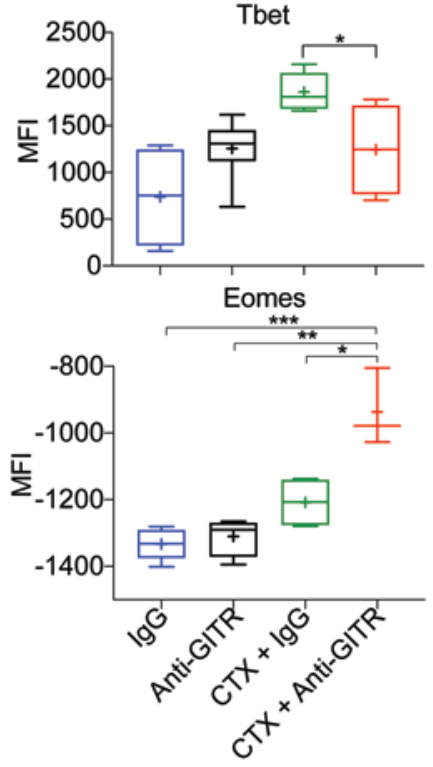

IFN $\gamma+$ TNF $\alpha+$

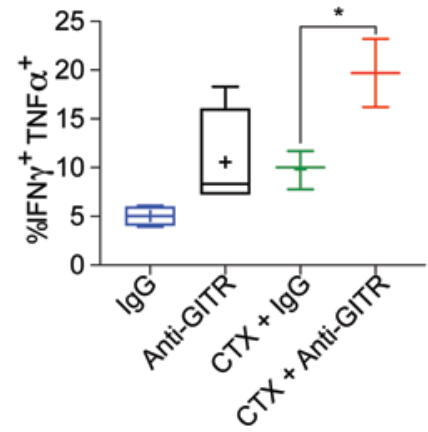

Figure 6. Combination of CTX and anti-GITR promotes effector $\mathrm{CD8}^{+} \mathrm{T}$ cell fitness. Cohorts of mice were implanted with B16. On day 8, CTX was injected. The next day, mice were treated with anti-GITR antibody or IgG. Additional mice were implanted with B16 on day 8 ; on day 15 mice were treated with anti-GITR or IgG. After 1 week, all cohorts of mice were sacrificed, and single-cell suspensions were prepared. Single-cell suspensions were stained with anti-CD5 $T$ cells and hashtag multiplex antibodies. The cells were FACS sorted for $\mathrm{CD}^{+}$ and next-generation TCR coupled with 10x Genomics sequencing. (A) Pearson correlations of $\mathrm{CD}^{+}$cells from scRNASeq between treatments (columns) and expression of predefined gene signatures demonstrating increased T cell activation in the combination-treated groups.

(B) Heatmap of selected cytolytic genes on $\mathrm{CD}^{+} \mathrm{T}$ cells. (C and $\left.\mathbf{D}\right)$ Mice were treated as described above, and 10 days after anti-GITR treatment single-cell suspensions were prepared from tumors. Cells were analyzed by flow cytometry. (C) MFI of PD-1, CD25, KLRG1, and Blimp-1 gated on $\mathrm{CD}^{+} \mathrm{T}$ cells. (D) Representative flow cytometry plots of T-bet versus Eomes on a $C D 8^{+} T$ cell gate (left); average MFI of T-bet and Eomes per treatment group (right). (E) Mice were treated as shown above and single-cell suspensions from tumors of mice were stimulated with PMA/ionomycin. After 6 hours, intracellular cytokine staining analysis was performed by flow cytometry. Representative flow plots (left). Percentage of TNF+IFN- $\gamma^{+}$per treatment (right). Box-and-whisker plots of 3-6 mice/group are shown where bounds of the box represent the 25 th and 75 th percentiles, the line represents the median, the plus sign represents the mean, and whiskers represent the minimum and maximum values. ${ }^{*} P<0.05,{ }^{*} P<0.01,{ }^{* *} P<0.001$. Flow cytometry experiments were repeated at least twice with similar results. 
Most of the expanded $\mathrm{CD}^{+} \mathrm{T}$ cell clones in the CTX + anti-GITR group clustered to a region in the UMAP visualized as a loop-like structure, which is indicative of active proliferation of these CD8 ${ }^{+}$ $\mathrm{T}$ effectors. Unsupervised clustering of a population in the CD8 cluster of the CTX + anti-GITR group that included 10 of the most overrepresented clones revealed numerous histone- and chromatin-associated transcripts, which indicated vigorous cell cycling. Indeed, we observed that Ki67, histones, and chromatinassociated transcripts were strongly expressed. The selected genes mapped exclusively to the "loop" region rich in $\mathrm{CD}^{+} \mathrm{T}$ cells from the CTX + anti-GITR group (Figure $8 \mathrm{C}$ ). To investigate this phenotype more closely, we generated a gene list and subsequent pathway analysis associated with the proliferation marker Ki67 and confirmed previous observations of highly cycling cell populations (Supplemental Figure 5 and Supplemental Table 3). Heatmap analysis of transcripts from cells derived from different treatments confirmed high gene expression levels exclusively in the CTX + anti-GITR group, indicating that combination treatment resulted in a highly proliferative $\mathrm{CD} 8^{+} \mathrm{T}$ cell burst (Figure $8 \mathrm{D}$ ).

It is conceivable that the phenotype and clonal expansion of T cell subsets in a bulk analysis of a pooled cohort of mice could be driven by nonrepresentative outliers. To address this, we labeled cell suspensions from individual mice with a "hashtag" antibody before pooling and cell sorting (51). After sequencing and analysis, cells derived from each mouse can be segregated in silico. The majority of clones and phenotypes were consistent between mice that received equivalent treatment (Supplemental Figures 4 and 5 and Supplemental Table 4). The top 5 clones in the CTX + anti-GITR group were in the highly activated region of $\mathrm{CD}^{+} \mathrm{T}$ cells in all 5 mice. Overall, these experimental approaches identified TCRs with a highly activated/cytolytic phenotype in mice treated with CTX + anti-GITR. Future experiments will determine the specificity and avidity of the clones that promoted potent antitumor immunity, which could facilitate engineering of $\mathrm{T}$ cells that can be incorporated in adoptive $\mathrm{T}$ cell transfer protocols.

\section{Discussion}

In this study, we rationally combined the alkylating chemotherapeutic CTX with GITR agonist antibodies, which led to potent and durable antitumor responses in several clinically relevant models, including MPC11 plasmacytoma and the poorly immunogenic B16 melanoma. We observed that CTX treatment was associated with the upregulation of GITR on the surface of T cell subsets in a dose-dependent manner. This treatment was also associated with preferential Treg depletion and Teff expansion. The expanded Teffs consisted of highly activated, tumor-infiltrating, oligoclonal CD8 ${ }^{+} \mathrm{T}$ cells.

Chemotherapy plus immunotherapy combinations are becoming increasingly common in clinical oncology practice, following recent FDA approvals in non-small cell lung cancer (52-54) and triplenegative breast cancer (55). Homeostatic proliferation of antigen-specific Teffs is an important outcome of chemotherapeutic interventions, such as CTX. CTX induces lymphopenia followed by homeostatic proliferation of tumor-specific Teffs (24-26). At high doses, CTX promotes profound lymphodepletion that can create immunological "space." During reconstitution from lymphopenia, T cells acquire an activated phenotype, upregulating molecules such as GITR, and tumor-reactive clones preferentially expand (22). This is a primary reason why high-dose CTX is included in conditioning regimens for adoptive cell transfer protocols and chimeric antigen receptor $\mathrm{T}$ cell therapy (56).

The mechanisms by which CTX acts in our combination therapy are likely multiple. Lymphodepletion on its own is insufficient to explain the efficacy because total-body irradiation at a dose that B16 is radioresistant to does not work in combination with anti-GITR. The data showing Pmel-1 antigen-specific $\mathrm{T}$ cell proliferation specifically in TDLNs of mice treated with CTX and anti-GITR support the hypothesis that CTX induces tumor death and release of the Pmel antigen for Pmel-1 T cell recognition. Further investigations are currently underway to examine why CTX, but not gem, which also slows tumor growth as a monotherapy and thus presumably elicits tumor cell death directly, uniquely combines with anti-GITR to augment tumor immunity.

An important mechanism by which tumors avoid immune elimination is the recruitment and activation of Foxp $3^{+}$Tregs, and the presence of these cells is an indicator of poor prognosis for patients with solid tumors $(4,5)$. Tregs suppress antitumor immune responses by a variety of mechanisms, including acting as an IL-2 sink to reduce the availability of IL-2 for effector cells and the secretion of immunosuppressive cytokines, such as TGF- $\beta$ and IL-10 (4). Highly suppressive Tregs are found in both mouse and human tumors, and a major focus is to develop strategies to mitigate the presence and function of these cells. Anti-CTLA-4 (57) and anti-CCR4 (58) are 2 such approaches, but neither have proved effective for the majority of patients. 
A
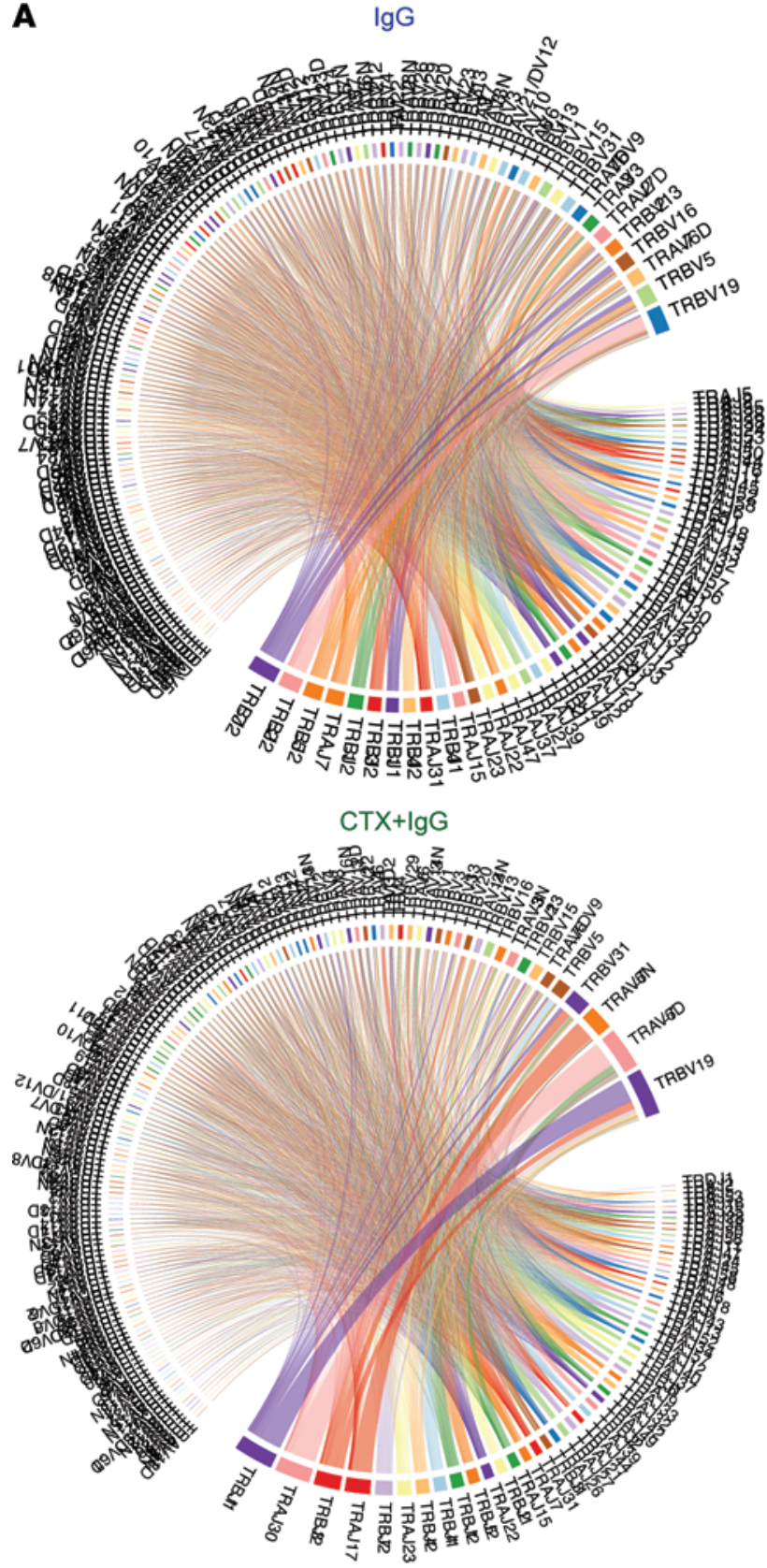

Anti-GITR

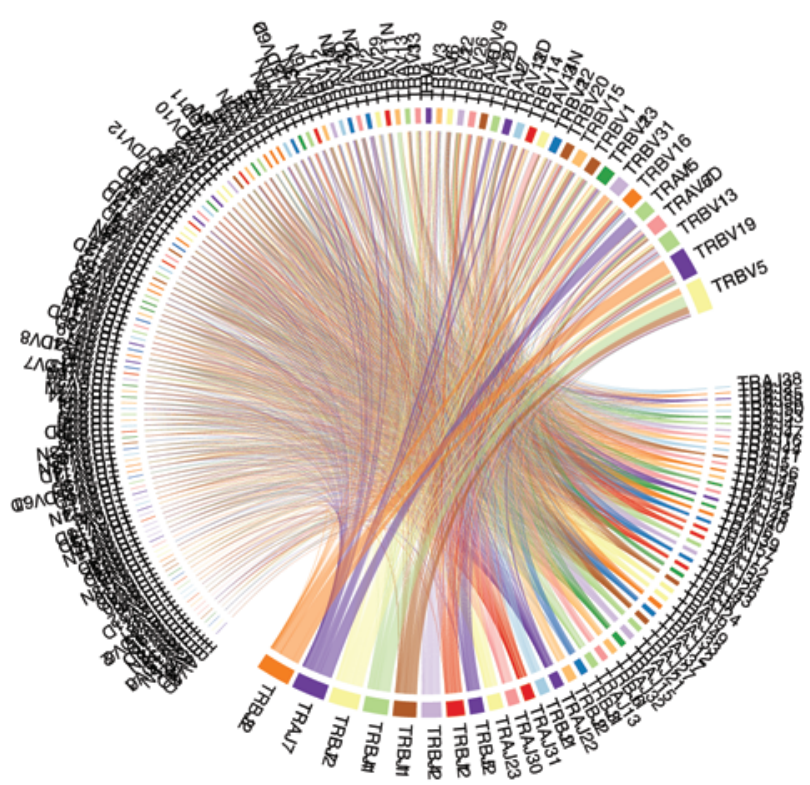

CTX+Anti-GITR

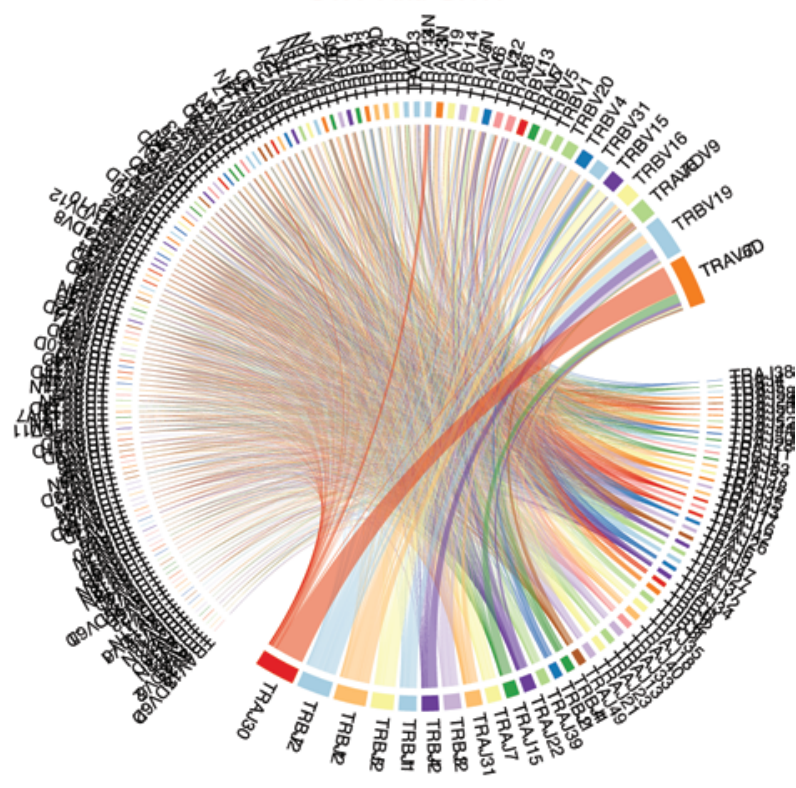

B

Shannon Weiner Evenness

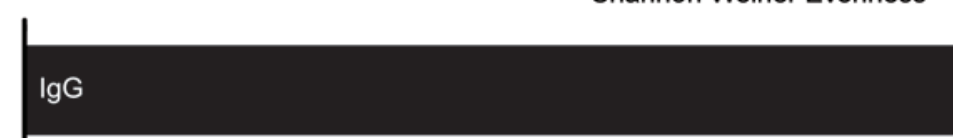

Anti-GITR

$\mathrm{CTX}+\lg \mathrm{Ig}$

CTX +Anti-GITR

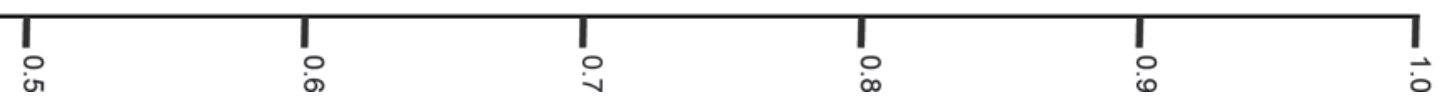


Figure 7. Combination therapy decreases the TCR repertoire. Cohorts of mice were implanted with B16. On day 8, CTX was injected. The next day, mice were treated with anti-GITR antibody or rat IgG. Additional cohorts of mice were injected with B16 on day 8; on day 15 mice were injected with anti-GITR or IgG. After 1 week, all cohorts of mice were sacrificed, and single-cell suspensions were prepared. Asynchronous B16 challenge was needed for the CTX groups a week apart given the lack of immune infiltrates caused by chemotherapy treatment. Single-cell suspensions were stained with anti-CD5 and hashtag multiplex antibodies. The cells were sorted by FACS based on $\mathrm{CDF}^{+}$, and next-generation TCR coupled with 10x Genomics sequencing was performed. (A) Circos plot displaying rearranged VDJ segments by treatment group, demonstrating an increased clonality in the combination-treated group. (B) Shannon-Weiner diversity evenness score for individual treatment groups.

Engaging GITR is an effective strategy to reduce or deactivate the suppressive function of Tregs and promote antitumor immunity. In this study, we demonstrated by both flow cytometry and scRNA-Seq that the combination of CTX and GITR agonist antibodies profoundly depletes intratumor Tregs. Closer examination revealed that Treg elimination occurred by induced cell death of hyperactivated Tregs. Interestingly, this is similar to the known cell death of Tregs observed in autoimmunity $(59,60)$. Other reports have described similar hyperactivation and potent antitumor immunity when engaging members of the TNF receptor family, such as OX40 $(61,62)$. The Tregs that failed to succumb to anti-GITR-induced cell death showed an increased Teff signature, characterized by upregulation of Teff and cytolytic markers. This observation is consistent with previous data from our laboratory showing that GITR agonism abolishes Treg suppressive function and destabilizes their phenotype to become effector like (7, 63). This "Teff-like" phenotype on Tregs stimulated with GITR agonist antibodies was more pronounced in cohorts of mice treated with CTX; perhaps the upregulation of surface GITR after CTX treatment underlies this observation (Figure 1). Future studies will establish if "Teff-like" Tregs possess actual antitumor functionality.

Previous mouse studies showed that GITR-induced depletion of Tregs is not sufficient to generate significant antitumor immunity, and effective engagement of GITR on Teffs is also needed $(64,65)$. Here we found that the combination of CTX and GITR agonism further promoted highly activated, cytolytic CD $8^{+}$ $\mathrm{T}$ cells. More specifically, $\mathrm{CD} 8^{+} \mathrm{T}$ cells isolated from tumors of mice treated with combination therapy showed downregulation of KLRG1, consistent with improved antitumor activity (66). We also observed upregulation of Blimp1 in CD8 ${ }^{+} \mathrm{T}$ cells, suggestive of a terminally differentiated state (67). Most notably, $\mathrm{CD}^{+} \mathrm{T}$ cells from mice treated with combination CTX + GITR agonist antibodies produced higher levels of TNF- $\alpha$ and IFN- $\gamma$. These cells were more activated and had a less dysfunctional phenotype, characterized by high levels of CD25 and low levels of PD-1. Our analysis also revealed that a subset of CD8 ${ }^{+} \mathrm{T}$ cells from tumors of mice treated with combination therapy showed upregulation of Eomes and downregulation of T-bet. It remains unclear whether this particular phenotype is associated with tumor growth or regression because results from prior work across different models are conflicting (45-47, 66, 67); nevertheless, Eomes seems to be expressed at the end stages of the cytolytic program (68). Therefore, in addition to the effects on clonal expansion of tumor antigen-specific T cells, our data indicate that combination therapy with CTX and GITR engagement promotes highly cytolytic, terminally differentiated $\mathrm{CD} 8^{+} \mathrm{T}$ cells.

Recent advances in sequencing technology enable the detailed study of TCR repertoires in response to immunotherapy (69). As a result, TCR diversity has been suggested as a prognostic biomarker for monitoring immune responses when evaluating novel immunotherapies (70-72). An open question remains as to whether diversification of TCR repertoires or clonal proliferation underlies favorable outcomes in immunotherapy. It stands to reason that better outcomes might be associated with a diverse starting TCR repertoire, which contains multiple clones targeting multiple antigens that can efficiently eliminate tumors and prevent antigen escape variants $(38,73-75)$. However, the response to treatment is expected to be associated with the expansion of a few high-affinity clones that possess robust effector functionality (37, 76-80). Our results showed that, indeed, a reduced TCR repertoire with expansion of highly activated clones was associated with control of tumor growth and/or regression. We demonstrated that combination therapy promoted clones with high expression of genes involved in cell cycling and mitosis. Among these genes are a series of histone and chromatin-associated proteins associated with a vigorous proliferative burst (81-83). Based on the immunomodulatory and tumor-cytotoxic effects of CTX and the stimulatory effects of GITR, it was not surprising that high-affinity TCR clones were expanded after the combination treatment. CTX-induced lymphodepletion and subsequent homeostatic proliferation can promote expansion of higher affinity TCR clones (84). We believe the TCR data are extremely useful for future work that can yield potential therapeutic benefit, and we are currently investigating the specificity of the expanded clones, examining whether they recognize shared or unique tumor antigens. Based on this, we will ultimately evaluate whether the TCRs specific for tumor tissuerestricted antigens might be adopted for engineering into adoptive cell therapy approaches. 
A

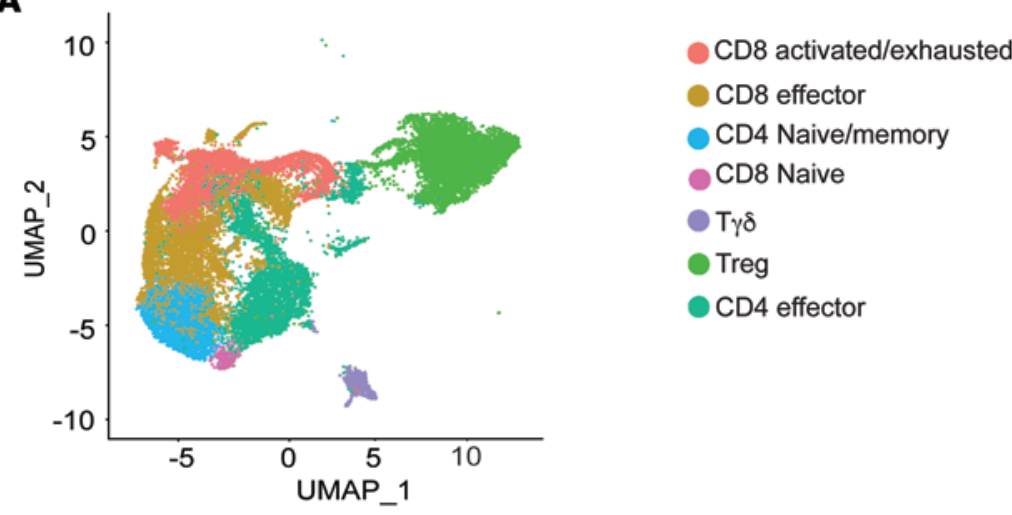

B

$\lg G$

Anti-GITR

CTX + IgG

CTX + Anti-GITR
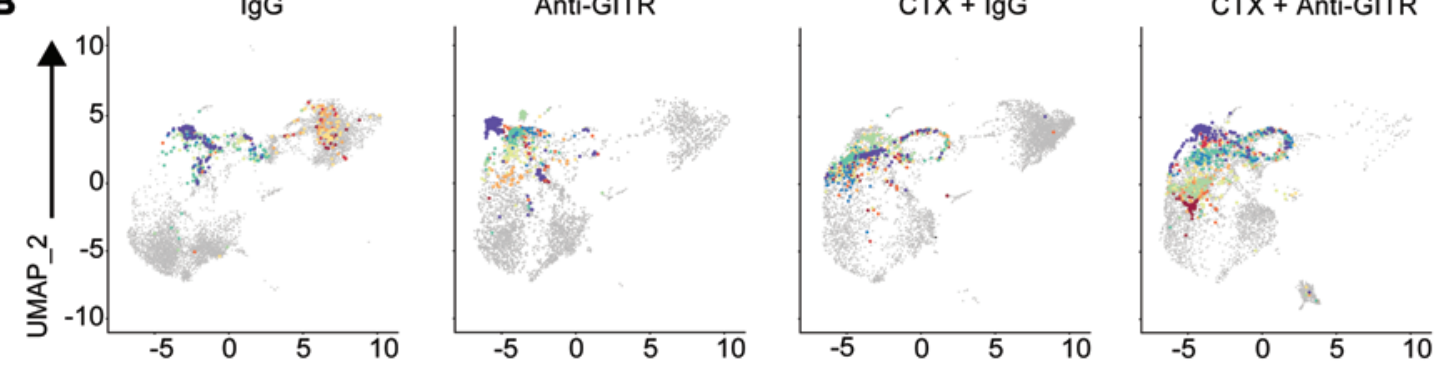

UMAP

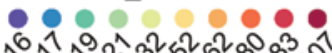
\#TCR clones

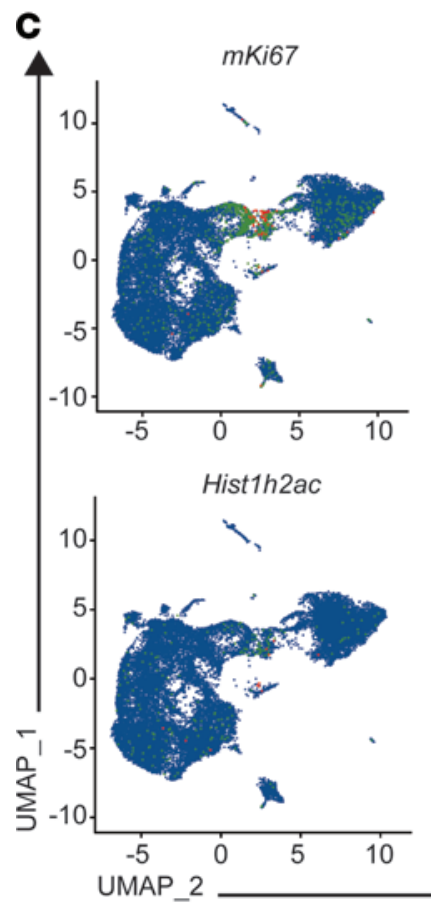

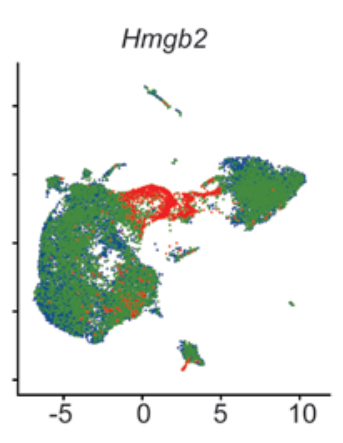

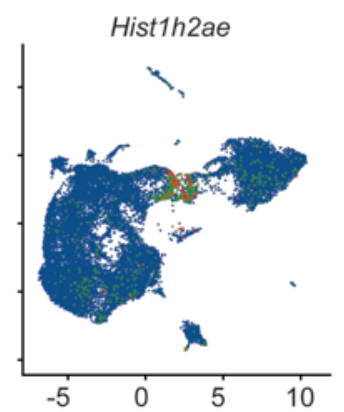

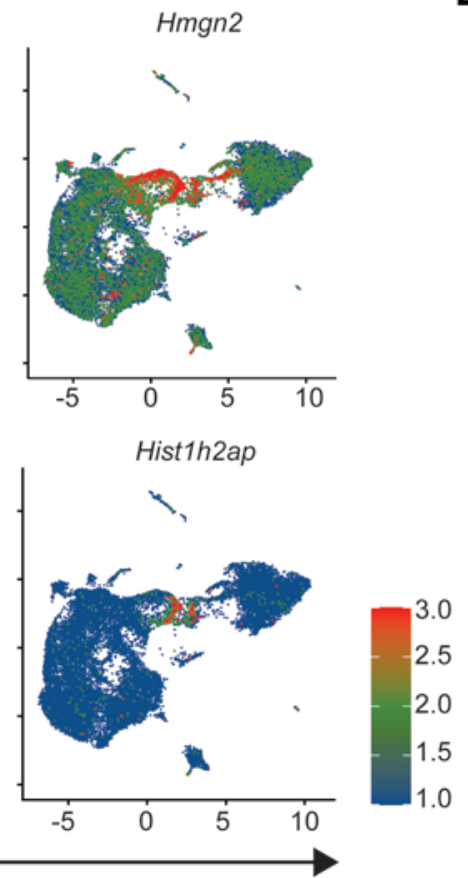

D
Histone Gene Expression (on CD8 Activated Cluster)

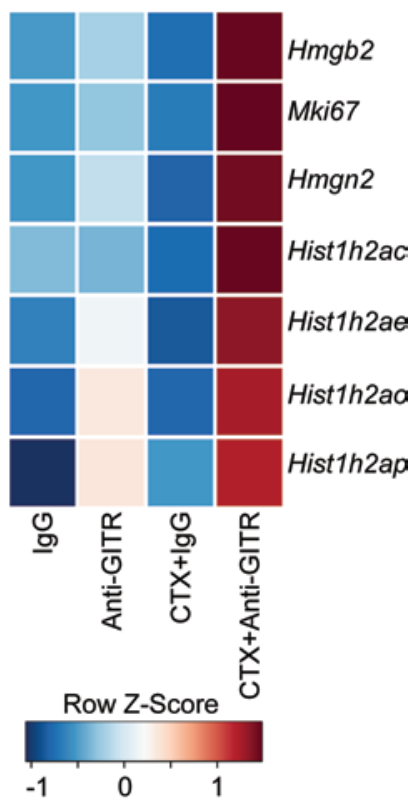

Figure 8. Combination therapy decreases the TCR repertoire by promoting clonal expansion of highly activated CD8 ${ }^{+} \mathbf{T}$ cells. Cohorts of mice were implanted with B16. On day 8, CTX was injected. The next day, mice were treated with anti-GITR antibody or rat IgG. Additional cohorts of mice were injected with B16 on day 8; on day 15 mice were injected with anti-GITR Ab or IgG. After 1 week, all cohorts of mice were sacrificed, and single-cell suspensions were prepared. Asynchronous B16 challenge was needed for the CTX groups a week apart given the lack of immune infiltrates caused by chemotherapy treatment. Single-cell suspensions were stained with anti-CD5 and hashtag multiplex antibodies. The cells were sorted by FACS based on CD5 ${ }^{+}$, and next-generation TCR coupled with 10x Genomics sequencing was performed. (A) UMAP displaying different clusters. (For a list of genes included in each subclusters refer to Supplemental Table 2.) (B) UMAP showing gene expression of the top 10 clones present in each treatment. Number below each colored circle represents the total number of cells in each clone. (For clone sequences refer to Supplemental Table 1.) (C) UMAP on selected cell cycle and proliferation genes. (D) Heatmap of selected cell cycle and proliferation genes per condition. 
Taken together, CTX and GITR agonism potently controlled tumor growth in several clinically relevant animal models. Mechanisms associated with the control of tumor growth included CTX-induced tumor cell death, clonal expansion of highly active and terminally differentiated $\mathrm{CD} 8^{+} \mathrm{T}$ cells, and depletion of Tregs by activation-induced cell death. The potency of this combination therapy in mice with poorly immunogenic tumors warrants further investigation in the clinical setting. Indeed, the combination of the anti-GITR antibody TRX-518 with CTX and PD-1 blockade was the focus of a phase Ib/IIa trial in immunotherapy-refractory solid tumors (NCT03861403), but data are not yet available on the safety or efficacy of the triplet combination.

\section{Methods}

Mice and tumor cell lines. C57BL/6J mice (6- to 8-week-old females) were obtained from The Jackson Laboratory. Pmel-1 TCR transgenic mice (Thy1.1) were originally a gift from N. Restifo (NIH, Bethesda, Maryland, USA) (41). All mice were bred at MSKCC. The B16-F10 (B16) mouse melanoma line was originally obtained from I. Fidler (MD Anderson Cancer Center, Houston, Texas, USA) and passaged intradermally in mice several times to ensure tumor growth. MPC-11 cells were obtained from ATCC. A lethal dose of $1 \times 10^{5} \mathrm{~B} 16$, and $5 \times 10^{5}$ MPC-11, cells was injected.

Monoclonal antibodies and drug treatment. Anti-GITR (DTA-1 clone) and IgG isotype control (rat IgG2b, anti-keyhole limpet hemocyanin) was obtained from Bio X Cell. For all treatments, $1 \mathrm{mg}$ anti-GITR or IgG was administered. Cyclophosphamide monohydrate (MilliporeSigma) mixed in sterile PBS was administered. Clinical-grade gem was obtained from the MSKCC Pharmacy and diluted in PBS to the appropriate dilution. Whole-body irradiation was given as a single dose of $6 \mathrm{~Gy}$. Both chemotherapies were administered as single doses intraperitoneally.

Antibodies, FACS analysis, and cell sorting. Antibodies used for flow cytometry analysis are listed in Supplemental Table 5 and were used according to the manufacturer's instructions with the recommended buffers. The Vybrant CFDA SE Cell Tracer kit (CFSE), DAPI, and the LIVE/DEAD Fixable Aqua Dead Cell Stain kit were obtained from Invitrogen and used according to the manufacturer's instructions. Sampled tumors were mechanically dissociated to obtain a single-cell suspension. Erythrocytes were removed using ACK lysing buffer (Invitrogen). Lymphocytes were clarified from tumors using 40\% Percoll (GE Healthcare) gradient centrifugation. Before staining, cells were treated with saturating anti-CD16/CD32 (BD) in staining buffer (2\% bovine serum albumin and $10 \mathrm{mM}$ EDTA in PBS) on ice for 15 minutes. Staining of surface antigens was performed in staining buffer on ice for 40 minutes. All intracellular staining was conducted using the Foxp3 Fixation/ Permeabilization Buffer (eBioscience) according to the manufacturer's instructions. For intracellular cytokine staining, single-cell suspension from tumors were incubated at $37^{\circ} \mathrm{C}$ with $20 \mathrm{ng} / \mathrm{mL}$ PMA (MilliporeSigma) and $1 \mu \mathrm{g} / \mathrm{mL}$ ionomycin (MilliporeSigma) in Happy T Cell Media (RPMI 1640 supplemented with 10\% FCS, $1 \times$ nonessential amino acids, $1 \mathrm{mM}$ sodium pyruvate, $2 \mathrm{mM}$ L-glutamine, and $50 \mu \mathrm{M} \beta$-mercaptoethanol) for 8 hours with $10 \mu \mathrm{g} / \mathrm{mL}$ monensin and $1 \mathrm{X}$ GolgiPlug (BD). Before staining, cells were treated with saturating anti-CD16/CD32 (BD) in staining buffer ( $2 \%$ bovine serum albumin and $2 \mathrm{mM}$ EDTA in PBS) on ice for 15 minutes. Staining of surface antigens was performed in staining buffer on ice for 40 minutes. All intracellular staining was conducted using BD Cytoperm/Cytofix reagents (BD). Flow cytometry was performed on a flow cytometer (LSRII; BD). FlowJo software (version 8.6.2; Tree Star, Inc.) was used for all flow cytometry analysis. FACS was conducted on a cell sorter (FACSDiva; BD).

Adoptive transfer experiments. Tumor-bearing mice received $2 \times 10^{6} \mathrm{CFSE}-1$ abeled CD8 $8^{+}$Pmel-1 cells intravenously via tail vein. $\mathrm{CD}^{+} \mathrm{T}$ cells were purified ( $<98 \%$ pure) from pooled spleens and lymph nodes from Pmel-1 mice (41) by positive selection using CD8a (Ly-2) microbeads (Miltenyi Biotec) according to the manufacturer's instructions. Purified cells were stained in PBS with $10 \mu \mathrm{M} \mathrm{CFSE}$ at $37^{\circ} \mathrm{C}$ for 10 minutes and quenched in complete media. The cells were washed twice in PBS before transfer. Five days later, single-cell suspensions were prepared from draining lymph nodes and analyzed by flow cytometry as described above.

scRNA-Seq. Cohorts of 5 mice were injected with B16. On day 8, CTX was injected. The next day, mice were injected with anti-GITR antibody or IgG. Additional cohorts of mice were injected with B16 on day 8; on day 15 mice were injected with DTA or IgG. After 1 week, all cohorts of mice were sacrificed, and single-cell suspensions were prepared. Asynchronous B16 challenge was needed for the CTX groups a week apart given the lack of immune infiltrates caused by chemotherapy treatment. Single-cell suspensions were simultaneously stained with anti-CD5 PE (BD) and TotalSeq anti-mouse hashtag antibodies (C0301, C0302, C0303, C0304, C0305; BioLegend). The cells were FACS sorted based on CD5 ${ }^{+}$, and next-generation TCR coupled with 10x Genomics sequencing was performed. 
scRNA-Seq data generation and processing. Single-cell sequencing data were aligned to the Genome Reference Consortium Mouse Build 38 (mm10) using Cell Ranger (v3.1.0; 10x Genomics) to obtain T cell clonotypes, feature barcoding, and gene expression profiles associated with individual single cells. Each data type was matched to create a unique molecular identifier matrix, and cells were filtered out based on 3 metrics: (i) cells with fewer than 200 detectable genes, (ii) cells with more than 2500 detectable genes, and (iii) cells that had less than $5 \%$ percentage of counts related to mitochondrial genes. Data normalization, principal component analysis, and subsequent UMAP were performed on the data set using R package Seurat v.3.1.1 (https://github.com/satijalab/seurat). The differential expression comparisons were generated using the DESeq2 package with selected genes (FDR < 0.05). t-Distributed stochastic neighbor embedding plots were constructed using PartekFlow software.

Supervised annotation of single cells. After filtering, we created subclusters of cells using the Louvain algorithm $(85,86)$. We classified each subcluster to a predefined cell type by performing a supervised analysis based on a list of known marker genes (CD8 activated: $C D 8 a^{+}, C D 8 b 1^{+} P_{v f 1^{+}}, N k g 7^{+}, \mathrm{Gzmb}^{+}, \mathrm{GzmK}^{+}, \mathrm{CD} 4^{-}$,

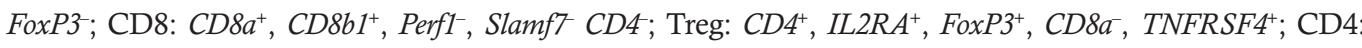
$C D 4^{+}, C D 8 a^{-}, C D 8 b^{-}, C D 28^{+}, F o x P 3-C D 8$ naive: $S_{e l l}^{+}, T c f 7^{+}, C D 8 a^{+}, C C R 7^{+}, C D 4 ;$ Tgd: $T c r g v 6^{+}, T r d v 4^{+}$, $I L 17 a^{+}, \operatorname{Trrg}^{-} \mathrm{Cl}^{+}, \mathrm{Trdc}^{+}, C D 8 a^{-}, C D 4$; CD4 naive: $C D 4^{+} \mathrm{Sell}^{+} \mathrm{FoxP3}^{-}, C C R 7^{+}$); $97 \%$ of the cells were distinctly annotated using this method, and the remaining cells were reviewed manually. We verified specific cell types using signature analysis for functional annotations $(87,88)$. Signature analysis per sample for a given subcluster was scored as the mean normalized expression across all genes listed for that signature.

TCR clonality. Clonality for each sample was calculated individually using Shannon-Weiner evenness. This was then compared across the different treatment conditions.

Data availability. Data presented in this study are available within the paper and its supplemental material. Publicly available data sets used in this study were available at National Center for Biotechnology Information Gene Expression Omnibus accession number GSE182292 (https://www.ncbi.nlm.nih.gov/geo/ query/acc.cgi?acc=GSE182292).

Statistics. All statistical analyses were calculated using GraphPad Prism software. Statistical differences between 2 experimental groups were determined by the unpaired 2-tailed Student's $t$ test. Statistical differences between more than 2 groups were calculated using a 1- or 2-way ANOVA followed by a post hoc Tukey's multiple comparisons test. Statistical differences between Kaplan-Meier survival curves were calculated using a log-rank (Mantel-Cox) test. The $\alpha$ value was set at 0.05 for all analyses, and $P$ values less than 0.05 were considered statistically significant.

Study approval. All mouse procedures were performed in accordance with the Institutional Animal Care and Use Committee guidelines at MSKCC under an approved protocol.

\section{Author contributions}

We determined the coauthorship by evaluating the key contribution of each author, and the first coauthorship was determined by the fact that the 2 first authors have been key contributors to the findings described in this manuscript. DH, ABW, TM, JDW, AC, ADC, SB, and ANH, GAR developed the concepts and discussed experiments. $\mathrm{DH}, \mathrm{ABW}, \mathrm{RM}, \mathrm{TM}$, and JDW wrote the manuscript. $\mathrm{DH}, \mathrm{ABW}, \mathrm{ADC}, \mathrm{SB}$, and NS performed and analyzed animal model experiments, flow cytometry experiments, and functional assays. DH, LMBM, and AC performed bioinformatics analysis. NS, LH, and CL provided technical assistance.

\section{Acknowledgments}

We thank K.J. Fitzgerald for her critical review of this manuscript. We thank the Flow Cytometry Facility and the Integrated Genomics Operation Core Facility at MSKCC. Swim Across America, Ludwig Institute for Cancer Research, Parker Institute for Cancer Immunotherapy, Center for Experimental Therapeutics at MSKCC, and the Breast Cancer Research Foundation supported this work. The work was also supported in part by R01 CA056821 (to JDW and TM) and the MSKCC Cancer Center Core Grant (P30 CA008748).

Address correspondence to: Jedd D. Wolchok or Taha Merghoub, 1275 York Avenue, New York, New York 10065, USA. Phone: 646.888.2315; Email: wolchokj@mskcc.org (JDW). Phone: 646.888.2580; Email: merghout@mskcc.org (TM). 
1. Lu J, et al. Clinical evaluation of compounds targeting PD-1/PD-L1 pathway for cancer immunotherapy. J Oncol Pharm Pract. 2015;21(6):451-467.

2. Yun S, et al. Targeting immune checkpoints in unresectable metastatic cutaneous melanoma: a systematic review and meta-analysis of anti-CTLA-4 and anti-PD-1 agents trials. Cancer Med. 2016;5(7):1481-1491.

3. Togashi Y, et al. Regulatory T cells in cancer immunosuppression - implications for anticancer therapy. Nat Rev Clin Oncol. 2019;16(6):356-371

4. Tanaka A, Sakaguchi S. Regulatory T cells in cancer immunotherapy. Cell Res. 2017;27(1):109-118.

5. Shang B, et al. Prognostic value of tumor-infiltrating FoxP3+ regulatory T cells in cancers: a systematic review and meta-analysis Sci Rep. 2015;5:15179.

6. Ronchetti S, et al. Role of GITR in activation response of T lymphocytes. Blood. 2002;100(1):350-352.

7. Schaer DA, et al. GITR pathway activation abrogates tumor immune suppression through loss of regulatory $\mathrm{T}$ cell lineage stability. Cancer Immunol Res. 2013;1(5):320-331.

8. Yates $\mathrm{K}$, et al. Comparative transcriptome analysis reveals distinct genetic modules associated with Helios expression in intratumoral regulatory T cells. Proc Natl Acad Sci U S A. 2018;115(9):2162-2167.

9. van Olffen RW, et al. GITR triggering induces expansion of both effector and regulatory CD4+ T cells in vivo. J Immunol. 2009;182(12):7490-7500.

10. Ronchetti S, et al. Glucocorticoid-induced tumour necrosis factor receptor-related protein: a key marker of functional regulatory T cells. J Immunol Res. 2015;2015:171520.

11. Ramirez-Montagut T, et al. Glucocorticoid-induced TNF receptor family related gene activation overcomes tolerance/ignorance to melanoma differentiation antigens and enhances antitumor immunity. J Immunol. 2006;176(11):6434-6442.

12. Ko K, et al. Treatment of advanced tumors with agonistic anti-GITR mAb and its effects on tumor-infiltrating Foxp3+CD25+CD4+ regulatory T cells. J Exp Med. 2005;202(7):885-891.

13. Shimizu J, et al. Stimulation of CD25(+)CD4(+) regulatory T cells through GITR breaks immunological self-tolerance. Nat Immunol. 2002;3(2):135-142.

14. Cohen $\mathrm{AD}$, et al. Agonist anti-GITR monoclonal antibody induces melanoma tumor immunity in mice by altering regulatory $\mathrm{T}$ cell stability and intra-tumor accumulation. PLoS One. 2010;5(5):e10436.

15. Koon HB, et al. First-in-human phase 1 single-dose study of TRX-518, an anti-human glucocorticoid-induced tumor necrosis factor receptor (GITR) monoclonal antibody in adults with advanced solid tumors. J Clin Oncol. 2016;34(15 suppl):3017.

16. Zappasodi R, et al. Rational design of anti-GITR-based combination immunotherapy. Nat Med. 2019;25(5):759-766.

17. Warner AB, Postow MA. Combination controversies: checkpoint inhibition alone or in combination for the treatment of melanoma? Oncology (Williston Park). 2018;32(5):228-234.

18. Fridman WH, et al. The immune contexture in human tumours: impact on clinical outcome. Nat Rev Cancer. 2012;12(4):298-306

19. Ghiringhelli F, et al. Metronomic cyclophosphamide regimen selectively depletes CD4+CD25+ regulatory T cells and restores $\mathrm{T}$ and NK effector functions in end stage cancer patients. Cancer Immunol Immunother. 2007;56(5):641-648.

20. Le DT, Jaffee EM. Regulatory T-cell modulation using cyclophosphamide in vaccine approaches: a current perspective. Cancer Res. 2012;72(14):3439-3444.

21. Suzuki E, et al. Gemcitabine selectively eliminates splenic Gr-1+/CD11b+ myeloid suppressor cells in tumor-bearing animals and enhances antitumor immune activity. Clin Cancer Res. 2005;11(18):6713-6721.

22. Brode S, Cooke A. Immune-potentiating effects of the chemotherapeutic drug cyclophosphamide. Crit Rev Immunol. 2008;28(2):109-126.

23. Nowak AK, et al. Induction of tumor cell apoptosis in vivo increases tumor antigen cross-presentation, cross-priming rather than cross-tolerizing host tumor-specific CD8 T cells. J Immunol. 2003;170(10):4905-4913.

24. Ghiringhelli F, et al. CD4+CD25+ regulatory T cells suppress tumor immunity but are sensitive to cyclophosphamide which allows immunotherapy of established tumors to be curative. Eur J Immunol. 2004;34(2):336-344

25. Ikezawa Y, et al. Cyclophosphamide decreases the number, percentage and the function of CD25+ CD4+ regulatory T cells, which suppress induction of contact hypersensitivity. J Dermatol Sci. 2005;39(2):105-112.

26. North RJ. Cyclophosphamide-facilitated adoptive immunotherapy of an established tumor depends on elimination of tumor-induced suppressor T cells. J Exp Med. 1982;155(4):1063-1074.

27. Bracci L, et al. Cyclophosphamide enhances the antitumor efficacy of adoptively transferred immune cells through the induction of cytokine expression, B-cell and T-cell homeostatic proliferation, and specific tumor infiltration. Clin Cancer Res. 2007;13(2 pt 1):644-653.

28. Schiavoni G, et al. Cyclophosphamide induces type I interferon and augments the number of CD44(hi) T lymphocytes in mice: implications for strategies of chemoimmunotherapy of cancer. Blood. 2000;95(6):2024-2030.

29. Anderson D, et al. Cyclophosphamide: review of its mutagenicity for an assessment of potential germ cell risks. Mutat Res. 1995;330(1):115-181.

30. Du B, Waxman DJ. Medium dose intermittent cyclophosphamide induces immunogenic cell death and cancer cell autonomous type I interferon production in glioma models. Cancer Lett. 2020;470:170-180.

31. McGranahan N, et al. Clonal neoantigens elicit T cell immunoreactivity and sensitivity to immune checkpoint blockade. Science. 2016;351(6280):1463-1469.

32. Hunter C, et al. A hypermutation phenotype and somatic MSH6 mutations in recurrent human malignant gliomas after alkylator chemotherapy. Cancer Res. 2006;66(8):3987.

33. Cahill DP, et al. Loss of the mismatch repair protein MSH6 in human glioblastomas is associated with tumor progression during temozolomide treatment. Clin Cancer Res. 2007;13(7):2038-2045.

34. Heather JM, et al. High-throughput sequencing of the T-cell receptor repertoire: pitfalls and opportunities. Brief Bioinform 2018;19(4):554-565.

35. Cha E, et al. Improved survival with T cell clonotype stability after anti-CTLA-4 treatment in cancer patients. Sci Transl Med. 2014;6(238):238ra70.

36. Roh W, et al. Integrated molecular analysis of tumor biopsies on sequential CTLA-4 and PD-1 blockade reveals markers of response and resistance. Sci Transl Med. 2017;9(379):eaah3560.

37. Tumeh PC, et al. PD-1 blockade induces responses by inhibiting adaptive immune resistance. Nature. 2014;515(7528):568-571 
38. Aversa I, et al. Molecular T-cell repertoire analysis as source of prognostic and predictive biomarkers for checkpoint blockade immunotherapy. Int J Mol Sci. 2020;21(7):E2378.

39. Trowell OA. The sensitivity of lymphocytes to ionising radiation. J Pathol Bacteriol. 1952;64(4):687-704.

40. Chen $\mathrm{H}-\mathrm{Y}$, et al. Inhibiting the $\mathrm{CD}^{+} \mathrm{T}$ cell infiltration in the tumor microenvironment after radiotherapy is an important mechanism of radioresistance. Sci Rep. 2018;8(1):11934.

41. Overwijk WW, et al. Tumor regression and autoimmunity after reversal of a functionally tolerant state of self-reactive CD8+ T cells. J Exp Med. 2003;198(4):569-580.

42. Turk MJ, et al. Concomitant tumor immunity to a poorly immunogenic melanoma is prevented by regulatory T cells. $J$ Exp Med 2004;200(6):771-782.

43. Quezada SA, et al. CTLA4 blockade and GM-CSF combination immunotherapy alters the intratumor balance of effector and regulatory T cells. J Clin Invest. 2006;116(7):1935-1945.

44. Guo X, et al. Global characterization of T cells in non-small-cell lung cancer by single-cell sequencing. Nat Med. 2018;24(7):978-985

45. Shin H, et al. A role for the transcriptional repressor Blimp-1 in CD8(+) T cell exhaustion during chronic viral infection. Immunity 2009;31(2):309-320.

46. Li J, et al. High levels of eomes promote exhaustion of anti-tumor CD8+ T cells. Front Immunol. 2018;9:2981.

47. Buggert M, et al. T-bet and Eomes are differentially linked to the exhausted phenotype of CD8+ T cells in HIV infection. PLoS Pathog. 2014;10(7):e1004251.

48. Cowell LG. The diagnostic, prognostic, and therapeutic potential of adaptive immune receptor repertoire profiling in cancer Cancer Res. 2020;80(4):643-654.

49. van der Leun AM, et al. CD8 ${ }^{+}$T cell states in human cancer: insights from single-cell analysis. Nat Rev Cancer. 2020;20(4):218-232.

50. Yu X, et al. Unravelling the heterogeneity and dynamic relationships of tumor-infiltrating T cells by single-cell RNA sequencing analysis. J Leukoc Biol. 2020;107(6):917-932.

51. Stoeckius M, et al. Cell Hashing with barcoded antibodies enables multiplexing and doublet detection for single cell genomics Genome Biol. 2018;19(1):224.

52. Gandhi L, et al. Pembrolizumab plus chemotherapy in metastatic non-small-cell lung cancer. N Engl J Med. 2018;378(22):2078-2092

53. Paz-Ares L, et al. Pembrolizumab plus chemotherapy for squamous non-small-cell lung cancer. NEngl J Med. 2018;379(21):2040-2051.

54. Socinski MA, et al. Atezolizumab for first-line treatment of metastatic nonsquamous NSCLC. N Engl J Med. 2018;378(24):2288-2301.

55. Schmid P, et al. Atezolizumab and Nab-paclitaxel in advanced triple-negative breast cancer. N Engl J Med. 2018;379(22):2108-2121

56. Brentjens RJ, et al. Safety and persistence of adoptively transferred autologous CD19-targeted T cells in patients with relapsed or chemotherapy refractory B-cell leukemias. Blood. 2011;118(18):4817-4828.

57. Simpson TR, et al. Fc-dependent depletion of tumor-infiltrating regulatory T cells co-defines the efficacy of anti-CTLA-4 therapy against melanoma. J Exp Med. 2013;210(9):1695-1710.

58. Ueda R. Clinical application of anti-CCR4 monoclonal antibody. Oncology. 2015;89(suppl 1):16-21.

59. Tang Q, et al. Central role of defective interleukin-2 production in the triggering of islet autoimmune destruction. Immunity. 2008;28(5):687-697.

60. Visperas A, Vignali DA. Are regulatory T cells defective in type 1 diabetes and can we fix them? J Immunol. 2016;197(10):3762-3770

61. Hirschhorn-Cymerman D, et al. OX40 engagement and chemotherapy combination provides potent antitumor immunity with concomitant regulatory T cell apoptosis. J Exp Med. 2009;206(5):1103-1116.

62. Schaer DA, et al. Targeting tumor-necrosis factor receptor pathways for tumor immunotherapy. J Immunother Cancer. $2014 ; 2: 7$.

63. Verma A, et al. T-regulatory cells in tumor progression and therapy. Cancer Manag Res. 2019;11:10731-10747.

64. Werfel TA, et al. Treatment-induced tumor cell apoptosis and secondary necrosis drive tumor progression in the residual tumor microenvironment through MerTK and IDO1. Cancer Res. 2019;79(1):171-182.

65. Kim YH, et al. Authentic GITR signaling fails to induce tumor regression unless Foxp3+ regulatory T cells are depleted. J Immunol. 2015;195(10):4721-4729.

66. Herndler-Brandstetter D, et al. KLRG1 ${ }^{+}$effector $\mathrm{CD} 8^{+} \mathrm{T}$ cells lose KLRG1, differentiate into all memory $\mathrm{T}$ cell lineages, and convey enhanced protective immunity. Immunity. 2018;48(4):716-729.

67. Rutishauser RL, et al. Transcriptional repressor Blimp-1 promotes CD8(+) T cell terminal differentiation and represses the acquisition of central memory T cell properties. Immunity. 2009;31(2):296-308.

68. Cruz-Guilloty F, et al. Runx3 and T-box proteins cooperate to establish the transcriptional program of effector CTLs. J Exp Med. 2009;206(1):51-59.

69. Jiang N, et al. Ushering in integrated T cell repertoire profiling in cancer. Trends Cancer. 2019;5(2):85-94.

70. Gambone JC, et al. Validation of hysterectomy indications and the quality assurance process. Obstet Gynecol. 1989;73(6):1045-1049.

71. Page DB, et al. Deep sequencing of T-cell receptor DNA as a biomarker of clonally expanded TILs in breast cancer after immunotherapy. Cancer Immunol Res. 2016;4(10):835-844.

72. Han Y, et al. Immune repertoire: a potential biomarker and therapeutic for hepatocellular carcinoma. Cancer Lett. 2016;379(2):206-212.

73. Kansy BA, et al. T cell receptor richness in peripheral blood increases after cetuximab therapy and correlates with therapeutic response. Oncoimmunology. 2018;7(11):e1494112.

74. Kwek SS, et al. Preexisting levels of CD4 T cells expressing PD-1 are related to overall survival in prostate cancer patients treated with ipilimumab. Cancer Immunol Res. 2015;3(9):1008-1016.

75. Robert L, et al. CTLA4 blockade broadens the peripheral T-cell receptor repertoire. Clin Cancer Res. 2014;20(9):2424-2432.

76. Park JH, et al. Clonal expansion of antitumor T cells in breast cancer correlates with response to neoadjuvant chemotherapy. Int $J$ Oncol. 2016;49(2):471-478.

77. Inoue $\mathrm{H}$, et al. Intratumoral expression levels of $P D-L 1, G Z M A$, and $H L A-A$ along with oligoclonal $\mathrm{T}$ cell expansion associate with response to nivolumab in metastatic melanoma. Oncoimmunology. 2016;5(9):e1204507.

78. Tamura K, et al. Characterization of the T cell repertoire by deep T cell receptor sequencing in tissues and blood from patients with advanced colorectal cancer. Oncol Lett. 2016;11(6):3643-3649.

79. Cooper ZA, et al. BRAF inhibition is associated with increased clonality in tumor-infiltrating lymphocytes. Oncoimmunology 2013;2(10):e26615. 
80. Snyder A, et al. Contribution of systemic and somatic factors to clinical response and resistance to PD-L1 blockade in urothelial cancer: an exploratory multi-omic analysis. PLoS Med. 2017;14(5):e1002309.

81. Best JA, et al. Transcriptional insights into the CD8(+) T cell response to infection and memory T cell formation. Nat Immunol. 2013;14(4):404-412.

82. Waugh KA, et al. Molecular profile of tumor-specific CD8+ T cell hypofunction in a transplantable murine cancer model. J Immunol. 2016;197(4):1477-1488.

83. Yost KE, et al. Clonal replacement of tumor-specific T cells following PD-1 blockade. Nat Med. 2019;25(8):1251-1259.

84. Surh CD, Sprent J. Homeostatic T cell proliferation: how far can T cells be activated to self-ligands? J Exp Med. 2000;192(4):F9-F14.

85. Blondel VD, et al. Fast unfolding of communities in large networks. J Stat Mech. 2008;(10):P10008.

86. Lancichinetti A, Fortunato S. Community detection algorithms: a comparative analysis. Phys Rev E Stat Nonlin Soft Matter Phys. 2009;80(5):056117.

87. Hemmers S, et al. IL-2 production by self-reactive CD4 thymocytes scales regulatory T cell generation in the thymus. $J$ Exp Med. 2019;216(11):2466-2478.

88. Singer M, et al. A distinct gene module for dysfunction uncoupled from activation in tumor-infiltrating T cells. Cell. 2016;166(6):1500-1511 